\title{
Optimal Fine and Medium Grain Parallelism Detection in Polyhedral Reduced Dependence Graphs ${ }^{1}$
}

\section{Alain Darte ${ }^{2}$ and Frédéric Vivien ${ }^{2}$}

\begin{abstract}
This paper presents an optimal algorithm for detecting line or medium grain parallelism in nested loops whose dependences are described by an approximation of distance vectors by polyhedra. In particular. this algorithm is optimal for the classical approximation by direction sectors. This result generalizes. to the case of several statements. Wolf and Lam's algorithm which is optimal for a single statement. Our algorithm relies on a dependence unitormization process and on parallelization techniques related to system of uniform recurrence equations. It can also be viewed as a combination of both Alkn and Kennedy's algorithm and Woll and Lam's algorithm.
\end{abstract}

KEY WORDS: Automatic parallelization: multi-dimensional schedule: loop nest: system of unilorm recurrence equations: dependence analysis: polyhedral reduced dependence graph.

\section{INTRODUCTION}

Loop transformations are useful source to source transformations for improving the performance of programs, for detecting parallelism, and for improving data locality. Year after year. a catalog of such transformations has been developed (see for example the survey paper by Bacon et al.'") but how to combine these transformations to achieve some particular goal remains a research topic. In the past ten years, many researchers have proposed various algorithms and frameworks that unify (some of) these

' Supported by the CNRS-INRIA project ReMaP.

Z Laboratoire LIP. URA CNRS 1398. Feole Nomale Supirieure de Lyon. F-69364 Lyon Cedex 07

447 
code transformations: Allen and Kennedy's algorithm ${ }^{(2)}$ for handling loop distribution and loop fusion, Banerjee's framework ${ }^{(3)}$ and Wolf and Lam's algorithm ${ }^{(4)}$ for handling unimodular transformations, i.e., combinations of loop reversal, loop skewing and loop interchange, Kelly and Pugh's framework $^{(5)}$ and Feautrier's algorithm for handling general affine transformations. ${ }^{(6)}$

Concerning this last framework, it is interesting to notice that fundamental differences exist between Kelly and Pugh's approach and Feautrier's approach: the first approach is a "try and test" heuristic, where transformations are selected and analyzed in terms of granularity, data locality, code complexity, while the second approach is a completely specified algorithm whose only goal is to build as many parallel inner loops as possible. Which approach is the best? Since none of them is implemented in a real compiler, we can only give theoretical motivations. The strength of Feautrier's approach is twofold: first, in a unified framework, it gives an upper bound on the maximal theoretical parallelism that can be detected by affine transformations, second its result can be reproduced by anybody since the algorithm is fully specified. This second advantage is also its weakness: it is not flexible enough to handle other objectives. For example, there is no guarantee concerning the simplicity of the generated code, and no clear way is given for increasing the granularity of the parallelized code. On the other hand, even though these criteria can be taken into account by Kelly and Pugh, they can miss a good solution found by Feautrier's algorithm because of their heuristic strategy.

In fact, Kelly and Pugh prefer a heuristic approach because parallelism detection is not the only issue. Among other criteria, simple code generation also is fundamental, and we believe that it is important to control the complexity of the transformations generated by a parallelizing algorithm. With this idea in mind, we present a parallelization algorithm, simpler than Feautrier's algorithm, which captures simultaneously loop distribution, index-shift method, and unimodular transformations. It combines techniques related to systems of uniform recurrence equations introduced by Karp et al., ${ }^{(7)}$ with the techniques involved in Allen and Kennedy's algorithm (Allen-Kennedy for short) and Wolf and Lam's algorithm (WolfLam for short). Our algorithm is in the "approximated class" as it takes as input an approximation of the dependences, called dependence polyhedra. Dependence polyhedra are a generalization of direction vectors: roughly speaking, dependence polyhedra are sort of direction vectors whose "directions" are not necessarily parallel to the axis of the basis. Thus, our algorithm can also work with classical direction vectors. In fact, each time we write "dependence polyhedra", the reader can read "direction vectors". We present here the more general version of our algorithm, the one which 
works on polyhedral reduced dependence graphs, as the generalization induces no overhead. Our algorithm has the following properties:

- It does not require an exact dependence analysis. It is optimal ${ }^{3}$ for dependence graphs whose edges are labeled by a polyhedral approximation of distance vectors. In particular, it is optimal for level of dependences and direction vectors. Actually, it behaves exactly as Allen-Kennedy when dependences are expressed by dependence levels. Furthermore, when dependences are expressed by direction vectors, it generalizes Wolf-Lam to the case of loop nests with multiple statements (Wolf-Lam is optimal if there is only one statement).

- It points out precisely which dependences prevent the parallelization or are responsible for a loss of parallelism. This property allows to better understand the link between the maximal degree of parallelism that can be detected and the accuracy of dependence abstractions. See Darte and Vivien ${ }^{(8)}$ for a complete study of this topic. This property also enables to use efficient techniques to remove disturbing false dependences (see Calland et al. ${ }^{(9)}$ ).

- By construction, it can be naturally adapted to the search for maximal sets of fully permutable loops which is, in theory, an equivalent problem, and is, in practice, a way to exploit medium-grain parallelism (for a complete study see Darte et al. ${ }^{(1(1)}$ ).

- It produces schedules as regular as possible in order to generate codes as simple as possible. Indeed, our algorithm rewrites the codes using affine schedules, but, unlike Feautrier's algorithm, these affine schedules are chosen such as as many statements as possible have the same linear part: the code generation can then be viewed as a sequence of partial unimodular transformations and loop distributions. As a result, our output codes are guaranteed to be simpler than Feautrier's codes. Moreover, the complexity of the algorithm itself is lower as demonstrated by the comparison study independently done at the University of Passau. ${ }^{\prime \prime \prime}$

- It is based on the resolutions of linear programs that are similar to those solved in Feautrier's algorithm. The only (through fundamental) difference is that we look for less general affine transformations. Despite this difference, our optimality result gives some hints concerning the optimality cases of Feautrier's algorithm that was first presented as a "greedy heuristic."

\footnotetext{
'All the mentioned optimality results are true with respert to the dependence andisis. This means that the algorithm is able to find all the parallelism contained in the representation of the dependences it takes as input.
} 
The rest of the paper is organized as follows. In Section 2, after a brief survey of existing parallelizing algorithms, we motivate our work by showing very simple (and real) code fragments for which neither Allen and Kennedy's algorithm, nor Wolf and Lam's algorithm, is able to detect some parallelism. In Section 3, we illustrate the use of a polyhedral approximation of the dependences. Then, we formally define polyhedral reduced dependence graphs, and we demonstrate the expressive power of this dependence abstraction. In Section 4, we give an overview of the different steps of the parallelization algorithm, for perfectly nested loops. We illustrate its capabilities on an ad-hoc example. The proofs of correctness and optimality of the algorithm are detailed in the next two sections, which form the heart of the paper. Section 5 is devoted to the problem of computability of the dependence graph and the study of its properties, Section 6 addresses the scheduling problem and the efficiency of our solution. These two sections are fundamental to completely explain why the algorithm actually works, and to make the paper self-contained. However, they can be skipped at first reading. In Section 7, we discuss some implementation strategies that enables to reduce the complexity of the algorithm. We run our algorithm on a few exampies to illustrate its power compared to existing algorithms. Finally, we briefly show how it can be extended to nonperfect loop nests, even though some work remains to be done in this area. We conclude in Section 8 .

\section{MOTIVATION}

We are interested in compilers which automatically translate sequential programs into parallel programs. We especially look at algorithms that parallelize loop nests. These algorithms follow two different approaches. In the first approach, called "exact", the algorithms work with an exact representation of the dependences. In the second approach, called "approximated", the algorithms work with a conservative approximation of these dependences. We first survey these two approaches, exhibiting their advantages and drawbacks.

\subsection{The Exact Approach}

In this first approach, the parallelization algorithms take as input an exact representation of the dependences. The main algorithms are those of Lamport. ${ }^{121}$ Darte and Robert, ${ }^{13.14)}$ Feautrier, ${ }^{(6.15)}$ and of Lim and Lam: ${ }^{(16)}$

Lamport $^{(2)}$ proposed the hyperplane method that applies to a set of perfectly nested loops whose dependences are all uniform (a perfect 
uniform loop nest). Such a loop nest with $d$ loops always contains $d-1$ degrees of parallelism. The parallelized code contains one outer sequential loop and $d-1$ inner parallel loops.

Darte and Robert ${ }^{13,14)}$ look for an affine schedule for each statement in the loop nest. All dependences need to be uniform, and a quite large linear system (obtained by the duality theorem of linear programming) has to be solved. The schedule is selected as the minimal latency schedule among all possible affine schedules. This algorithm is optimal in terms of parallelism extraction: the latency of the selected schedule and the latency of the best existing schedule are guaranteed to be asymptotically equivalent ${ }^{(17.18)}$ for strongly connected dependence graphs. The technique can be extended to affine dependences (i.e., dependences expressed as affine functions of loop counters) but the optimality is not guaranteed any longer.

Feautrier ${ }^{16.15\}}$ has proposed a technique similar to Darte and Robert's technique for the one-dimensional case, except that the linear program is obtained by the affine form of Farkas' lemma. However, Feautrier's algorithm is more general since it is able to derive multi-dimensional affine schedules when no one-dimensional schedule exists. So far, Feautrier's algorithm is indeed the most powerful algorithm for inner parallelism detection in nested loops, even if, until now, it can be applied only to static control programs (see Feautier ${ }^{(19)}$ for a definition). There are some attempts to generalize this approach by weakening the constraints on the input, using a Fuzzy Array Dataflow Analysis. ${ }^{(20)}$ The only known results ${ }^{(17.18)}$ concerning the efficiency of this algorithm are the optimality cases of Darte and Robert's algorithm. However, there are no known results for the multi-dimensional case. Actually, what we call "Feautrier's algorithm" is named "greedy heuristic" by its author. ${ }^{(6)}$

Lim and Lam's $\mathbf{s}^{101}$ algorithm is a very recent extension of Feautrier's algorithm which finds fully permutable loops and external parallel loops (if any). The technique involves the affine form of Farkas' lemma and a variation of Fourier-Motzkin elimination. This algorithm is also devoted to programs where the array accesses are affine functions, but unlike the earlier three algorithms, the goal is not to look for the transformation which finds the maximal degree of parallelism, but to look for the affine transformation, if any, which finds a given degree of parallelism while minimizing the degree of synchronization. Their algorithm is said optimal under some hypotheses (but optimality has a different meaning).

We call "exact" the approach used in these four algorithms because they all rely on an exact dependence analysis and an exact representation of the dependences. The main advantage of this approach is that the loss of parallelism is never due to the computation of the dependences. 
However, some parallelism can be lost because of the parallelization algorithms themselves, and therefore their efficiency must be considered. Darte and Robert's algorithm, and in some cases Feautrier's algorithm, are optimal. But they are only optimal for the classes of programs they are able to process! All these algorithms require that exact dependence analysis is feasible and that dependences are affine. This implies very strong hypotheses on the original codes. Only programs with static control flow and affine array accesses can be processed. And not all algorithms previously given will process all of them. The limitation on the algorithms' inputs is the main drawback of this approach.

The exact approach is sometimes said to have another disadvantage: its cost. Indeed, the exact computation of the dependences is often said to be expensive. This can be discussed. What is sure however is that the execution time of Feautrier's algorithm, for example, is long as it requires the resolution of large linear programs (see for example ${ }^{\prime \prime \prime}$ for timing reports). Finally and, in our view, more important, the code generated by Feautrier's algorithm can be very complicated. Feautrier builds one multidimensional affine schedule per statement in the loop nest. This enables him to find schedules with theoretically efficient latencies... but the price to pay is the complexity of the code that may induce a loss of efficiency at run-time.

In conclusion, with the "exact" approach, no parallelism is lost because of the dependences representation. But there are strong requirements on the program to be parallelized. The efficiency of these parallelization algorithms is not known in the most general cases. Furthermore, most of them are computation expensive and the generated codes are complicated. Thus, the dream is to find a parallelization algorithm which handles more general programs and produces quickly simpler outputs, while having sufficient guarantees on its efficiency,

\subsection{The Approximated Approach}

In this approach, the parallelization algorithms take as input a conservative approximation of the dependences. The two main algorithms in this category are those of Allen and Kennedy, ${ }^{(2)}$ and Wolf and Lam: ${ }^{(4)}$

Allen and Kennedy ${ }^{(2)}$ proposed an algorithm based on loop distributions which are done by an analysis of the strongly connected components of the reduced dependence graph (RDG). Dependences are represented by levels, which is a very rough representation, although often sufficient on real codes. Allen and Kennedy's algorithm is known to be optimal ${ }^{(21)}$ with respect to its input. This means that a parallelization algorithm, which takes as input a reduced dependence graph where the dependences are 
represented by levels, cannot find more parallelism than Allen and Kennedy's algorithm does.

Wolf and $\mathbf{L a m}^{(+)}$algorithm is based on a cone separation technique, adapted to the case of direction vectors, and used to detect fully permutable loops. A set of $d$ fully permutable loops can always be rewritten as one sequential and $d-1$ parallel loops. The transformations used in Wolf-Lam are unimodular transformations. The representation of the dependences, by direction vectors, is sharper than in Allen-Kennedy. However, the graph structure of the dependences is not taken into account. Wolf-Lam is optimal ${ }^{(4)}$ among unimodular transformations which take direction vectors as input.

Unlike the "exact" approach, the "approximated" approach is able to process more general loop nests. This is its main advantage. For example, the parallelization algorithms in this category are able to handle codes containing array accesses with indirections: $A[B[I]]$. Another advantage is that they involve simpler techniques than the algorithms in the "exact" approach. Consequently, they are easier to implement. they are quicker and they generate simpler outputs. Furthermore, they have been proved optimal with respect to the representation of the dependences they used (see Refs. 21 and 22).

The problem of this approach is the cost, in terms of loss of parallelism, of an approximation of the dependences. Indeed, the dependences are conservatively approximated (over-approximated): the chosen dependence abstraction may represent more dependences than there are in the code. The additional dependences induce a greater apparent sequentiality of the original code, and this may cause a loss of parallelism. The problem is to determine whether this loss of parallelism is affordable. The fundamental question is: if the parallelization algorithm I used finds no parallelism in the loop nest, would another technique find some? Right now, there is no complete answer to this general question. However, a comparison of Allen-Kennedy and Wolf-Lam gives some hints.

\subsection{Allen-Kennedy vs. Wolf-Lam}

None of these two algorithms subsumes the other and both are sometimes unable to find parallelism in cases where there is obviously some. We illustrate these points with three real fragments of code, borrowed from Refs. 23 and 24. We also explain why combining loop distribution with the techniques involved in Wolf-Lam is not straightforward.

Example 1. (where Wolf-Lam beats Allen-Kennedy) Example 1, depicted in Fig. 1, is the Gauss-Seidel iteration program. ${ }^{(23)}$ There is in the 
dependence graph, Fig. 2, a dependence cycle at depth 1 and at depth 2 . Thus Allen-Kennedy finds no parallelism in this code. However, the dependences are uniform and equal to $(1,0)$ and $(0,1)$. Thus, Wolf-Lam finds that the two loops are fully permutable and that this loop nest contains some parallelism. This is the typical example where an algorithm such as Allen-Kennedy fails.

Example 2. (where Allen-Kennedy beats Wolf-Lam) Example 2, depicted in Fig. 3, is part of a linear equation solver based on matrix-vector operations, written by Dongarra and Eisenstat. ${ }^{(24)}$ Wolf-Lam algorithm, as described by Wolf and Lam, ${ }^{(4)}$ is only able to process perfectly nested loop nests. Thus, we first transform the code into a perfect one, before applying Wolf-Lam. There are many different solutions to this "perfectization" problem. One of them is given on Fig. 4.

The corresponding reduced dependence graph, computed by Petit [see Kelly et $\left.a l^{(25)}\right]$ (but in which redundant direction vectors have been removed), is drawn on Fig. 5. Because of the direction vectors of weight $(1,0-)$ and $(0,+)$, among others, Wolf-Lam is not able to detect any parallelism. However, there is clearly no cycle at depth 2 in the dependence graph. Thus, with a simple loop distribution at depth 2, Allen-Kennedy finds that the second loop can be made parallel. Actually, Allen-Kennedy is able to retrieve the original nonperfect version, with a parallel $i$ loop: running Allen-Kennedy on the original code would lead to the same parallelized code.

In this example, Wolf-Lam can be easily combined with Allen-Kennedy as follows. Instead of completely ignoring the structure of the dependence graph, we can apply a loop distribution before each recursive call to WolfLam. We can look for the first set of fully permutable loops, then apply loop distribution, then call Wolf-Lam again on the remaining dependences, etc. On this example, such a strategy enables us to find some parallelism when the original Wolf-Lam algorithm fails. However, there are examples where this simple modification is not sufficient to detect maximal parallelism (see Example 6 in Section 7.2).

Example 3. (where Wolf-Lam needs a perfect loop nest) For parallelizing Example 2, we first transformed the original code into a perfect code. One could argue that Wolf-Lam was not able to detect parallelism because of this transformation, and that Wolf-Lam should be apply on nonperfectly nested loops "set of perfectly nested loops" by "set of perfectly nested loops". Indeed, on Example 2, this modified version of WolfLam finds some parallelism. The first set of loops only includes the $k$ loop, which is detected as sequential. Then, the second set of loops only includes 


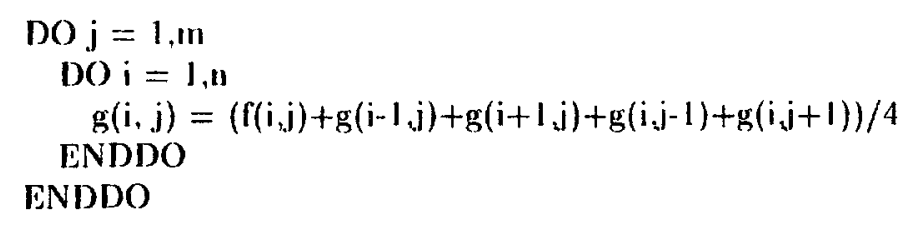

Hig. 1. Example 1. Gauss Scidel.

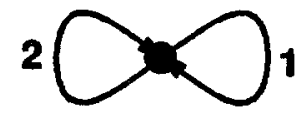

Fig. 2. RDG. Example 1.

$$
\begin{aligned}
& \text { DO } k=1, n \\
& S_{1}: x k=x(k) \times a(k, k) \\
& \text { DO } i=k+1, n \\
& S_{2}: x(i)=x(i)-a(i, k) \times x k \\
& \text { ENDDO } \\
& S_{3}: x(k)=x k \\
& \text { ENDDO }
\end{aligned}
$$

Fig. 3. Example 2. Dongarra Eisenstat.

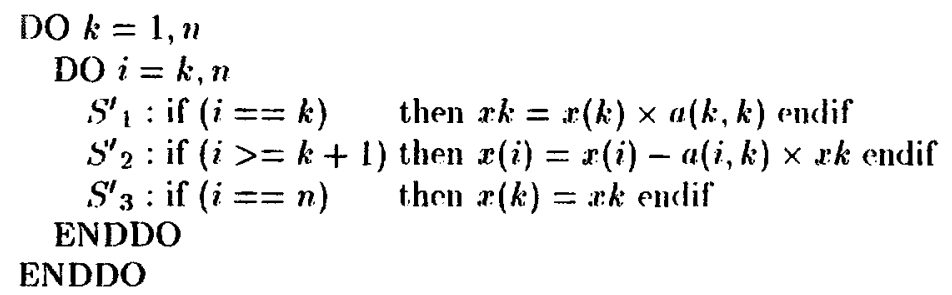

Fig. 4. A perfectly nested version of Example 2.

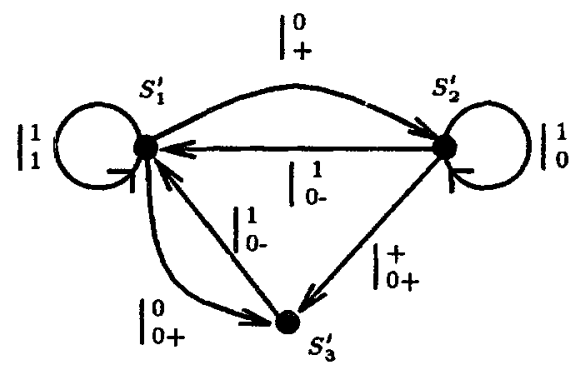

Fig. 5. Dependence graph of Dongarra and Eisenstat's perfectly nested version (Example 2). 


$$
\begin{aligned}
& \text { DO } k=0, n-1 \\
& \text { DO } i=n-k, n \\
& b(i)=b(i) /(x(i)-x(i-n+k)) \\
& \text { ENDDO } \\
& \text { DO } i=n-k-1, n-1 \\
& b(i)=b(i)-b(i+1) \\
& \text { ENDDO } \\
& \text { ENDDO }
\end{aligned}
$$

Fig. 6. Example 3. Vandermonde kernel.

the $i$ loop and the statement $S_{2}$. The $i$ loop contains no dependences and then is found parallel. Thus, in this case, it was not a good idea to transform the original nonperfect loop nest into a perfect one, before applying Wolf-Lam. However, Example 3 shows that this is sometimes a good idea.

Example 3 (see Fig. 6) is part of an algorithm to solve the Vandermonde system: $V z=b .^{(23)}$ Let us try to apply Wolf-Lam "set of perfectly nested loops" by "set of perfectly nested loops". The first set of perfectly nested loops only includes the $k$ loop. This loop is obviously sequential as $b(i)$ is written at several different $k$ iterations. For the second level, we have two sets of perfectly nested loops to look at. The first $i$ loop is parallel, as it contains no dependences. The second $i$ loop is sequential as the new value of $b(i)$ depends upon the old values of $b(i+1)$. Thus the program parallelized this way still contains two sequential nested loops as in the original program. Allen-Kennedy finds the same parallelized code. However, consider a perfectly nested version of this code as shown in Fig. 7. The direction vectors are $(+, 0),(0,1),(1,0),(+, 1),(+,-1)$, and $(1,-1)$. They are of course lexicographically positive. Furthermore their second

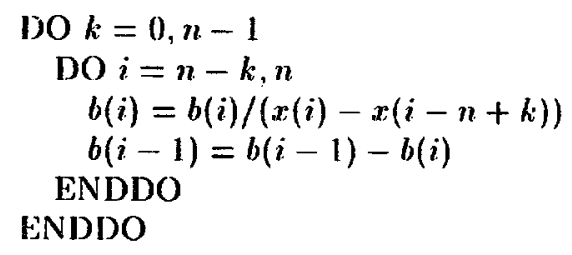

Fig. 7. A perfectly nested version of Example 3. 
component is always constant. Consequently, Wolf- Lam is able to find two permutable loops and, thus, one degree of parallelism. Therefore, on this example, transforming the code into perfectly nested loops and applying Wolf-Lam enables us to find more parallelism.

In conclusion, these three examples show that sometimes Wolf-Lam algorithm finds some parallelism when Allen-Kennedy only finds sequentiality, and vice versa. These examples also demonstrate that the basic Wolf-Lam algorithm needs to be upgraded to handle loop distribution. We proposed two new versions of Wolf-Lam in which we tried to combine loop distribution techniques as in Allen-Kennedy. We have now four different algorithms and no one subsumes the others. This confusing situation illustrates that combining unimodular transformations and loop distribution is not straightforward.

\subsection{Our Goal}

Schematically, we want an algorithm which works with an approximation of the dependences as we need to be able to process general loop nests; we want to use techniques powerful enough to extract all the parallelism described by the chosen representation of the dependences; we want to generate codes as "simple" as possible. We would like this algorithm to be able to detect some parallelism at least when Allen-Kennedy or Wolf-Lam does. Of course, such an algorithm exists: the algorithm which tries AllenKennedy and the different versions of Wolf-Lam and takes the "best" answer! The way this algorithm is built shows its main weakness: it uses either the dependence graph structure (Allen-Kennedy) or direction vectors (Wolf-Lam), but never knowledge of both types at the same time. For example, the simple Wolf-Lam extensions that we proposed earlier use the dependence graph structure before or after the computation of a maximal set of fully permutable loops, but never during this computation. The aim of Wolf and Lam ${ }^{(4)}$ was to "combine the mathematical rigor in the matrix transformation model with the generality of the vectorizing and concurrentizing compiler approach". We have the same goal, except that we would also like to exploit the structure of the dependence graph.

There is a large gap between the complexity of the algorithms in the "approximated" and "exact" approaches, both in terms of dependence abstraction and in terms of execution time. One of our goals is to fill this gap and to propose an intermediate algorithm, thus of medium complexity, but still optimal for all classical approximations of dependences. Moreover, 
we would like to generate "simple" codes, not to loose at running time what we theoretically gained using a more powerful parallelization algorithm. Thus, we want to use transformation techniques intermediate between those used by Allen-Kennedy and Wolf-Lam, which generate very simple codes, and those used by Feautrier's algorithm, which are far more expressive but can generate complicated codes.

\section{POLYHEDRAL APPROXIMATIONS OF DEPENDENCES}

\subsection{A Motivating Example}

We first introduce the notion of polyhedral reduced dependence graph (PRDG) on an ad-hoc example. PRDGs will be formally defined in Section 3.2. We show that no parallelism can be detected on this example if dependences are approximated by levels or direction vectors, but that an exact representation of dependences is not needed: an approximation of distance vectors by polyhedra is sufficient. We illustrate the underlying idea of our parallelizing algorithm, the "uniformization" principle, that enables us to capture PRDGs as if they were uniform dependence graphs.

Example 4. (use of polyhedral approximations) Example 4 is given in Fig. 8. The exact dependences are listed in Fig. 9. Figure 10 shows the corresponding (reduced) dependence graphs (RDGs) when dependence edges are labeled respectively with (a) levels and (b) direction vectors. Let us try to parallelize Example 4 with the algorithms described in Section 2: the algorithms of Allen and Kennedy; Wolf and Lam; Darte and Robert; and Feautrier.

- Allen-Kennedy The levels of the three dependences are respectively 2,1 , and 1 . There is a dependence cycle at depth 1 and at depth 2 . Therefore, no parallelism is detected.

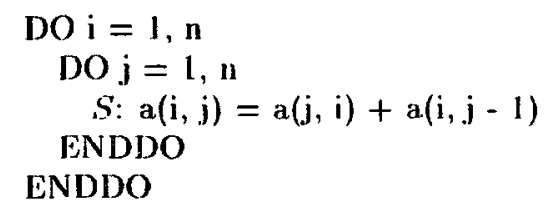

Fig. 8. Source code. 


$$
\begin{cases}\text { if } 1 \leq i \leq n, 1 \leq j<n & S(i, j) \stackrel{\text { flow }}{\longrightarrow} S(i, j+1) \\ \text { if } 1 \leq i<j \leq n & S(i, j) \stackrel{\text { flow }}{\longrightarrow} S(j, i) \\ \text { if } 1 \leq j<i \leq n & S(j, i) \stackrel{\text { anti }}{\longrightarrow} S(i, j)\end{cases}
$$

Fïg. 9. Exact dependence relations.

- Wolf-Lam The dependence vectors are respectively $(0,1),(+,-)$, and $(+,-)$. In the second dimension, the " 1 " and the "- prevent to detect two fully permutable loops. Therelore. the code remains unchanged, and no parallelism is detected.

- Darte-Robert One level of parallelism is detected with the valid linear schedule $T(i, j)=2 i+j$.

- Feautrier This algorithm finds the same schedule than Darte-Robert (up to a constant): $T(i, j)=2 i+j-3$. One level of parallelism is detected.

In this particular example. the representation of the dependences by levels or by direction vectors is not accurate enough to reveal parallelism. This is the reason why Allen-Kennedy and Wolf-Lam are not able to detect any parallelism. Exact dependence analysis, associated to linear programming methods that require to solve large ${ }^{4}$ parametric linear programs, enables to reveal one degree of parallelism. The corresponding parallelized code is given in Fig. 11.

However, in Example 4, an exact representation of the dependences is not necessary to reveal some parallelism. Indeed, one can notice that there is one uniform dependence $u=(0,1)$ and a set of distance vectors $\{(j-i, i-j)=(j-i)(1,-1) \mid 1 \leqslant j-i \leqslant n-1\}$ that can be (over)-approximated by the set $P=\{(1,-1)+\lambda(1,-1) \mid \lambda \geqslant 0\} . P$ is a polyhedron with one vertex $v=(1,-1)$ and one ray $r=(1,-1)$. Now, suppose that, as in the "exact" algorithms, we are looking for a linear schedule $T(i, j)=$ $x_{1} i+x_{2} j$. Let $X=\left(x_{1}, x_{2}\right)$. For $T$ to be a valid schedule, we look for $X$ such that $X d \geqslant 1$ for any dependence vector $d$. Thus, $X(0,1) \geqslant 1$ and $X p \geqslant 1$ for all $p \in P$. The latter inequality is equal to: $X(1,-1)+\lambda X(1,-1) \geqslant 1$ with $\lambda \geqslant 0$, which is equivalent to: $X(1,-1) \geqslant 1$ and $X(1,-1) \geqslant 0$, i.e.,

\footnotetext{
The number of inequalities and variables is related to the number of constraints that define the validity domain of each dependence relation.
} 
(a)

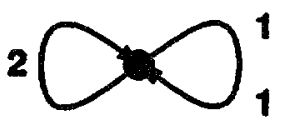

(b)

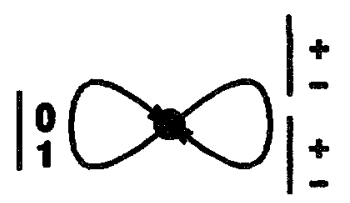

Fig. 10. RDG for Example 4.

$X v \geqslant 1$ and $X r \geqslant 0$. Therefore, one has just to solve the three following inequalities:

$$
X u \geqslant 1 \quad X v \geqslant 1 \quad X r \geqslant 0 \text { i.e., } \quad X\left(\begin{array}{l}
0 \\
1
\end{array}\right) \geqslant 1 \quad X\left(\begin{array}{r}
1 \\
-1
\end{array}\right) \geqslant 1 \quad X\left(\begin{array}{r}
1 \\
-1
\end{array}\right) \geqslant 0
$$

which leads, as before, to $X=(2,1)$. Thus, for this example, an approximation of the dependences by levels or even direction vectors is not sufficient for the detection of parallelism. However, with an approximation of the dependences by polyhedra, we find the same parallelism as with exact dependence analysis, by solving a simpler set of inequalities.

What is important here is the "uniformization" which enables us to go from the inequality on the set $P$ to uniform inequalities on $v$ and $r$. Thanks to this uniformization, the affine constraints disappear and unlike Feautrier's algorithm, we do not need to use Farkas' lemma. To better understand the "uniformization" principle, think in terms of dependence path. The idea is to consider an edge $e$, from statement $S$ to statement $T$, labeled by the distance vector $p=v+\lambda r$, as a path $\phi$ that uses once the "uniform" dependence vector $v$ and $\lambda$ times the "uniform" dependence vector $r$. This simulation is summarized in Fig. 12: we introduce a new node $S^{\prime}$ that enables to simulate $\phi$ and a zero weight edge to go from $S^{\prime}$ back to the initial node $T$. This "uniformization" principle is the underlying idea of the loop parallelization algorithm proposed in this paper.

By uniformizing the dependences, we have in fact "uniformized" the constraints and transformed the underlying affine scheduling problem into a simple scheduling problem where all dependences are uniform $(0, v$, and

$$
\begin{aligned}
& \text { DO } j=3,3 n \\
& \text { DOPAR } i=\max \left(1,\left[\frac{j-n}{2}\right\rceil\right), \min \left(n,\left\lfloor\frac{j-1}{2}\right\rfloor\right) \\
& \quad a(i, j-2 i)=\mathrm{a}(j-2 i, i)+\mathrm{a}(i, j-2 i-1) \\
& \text { ENDDO } \\
& \text { ENDDO }
\end{aligned}
$$

Fig. 11. Example 4. parallelized version. 


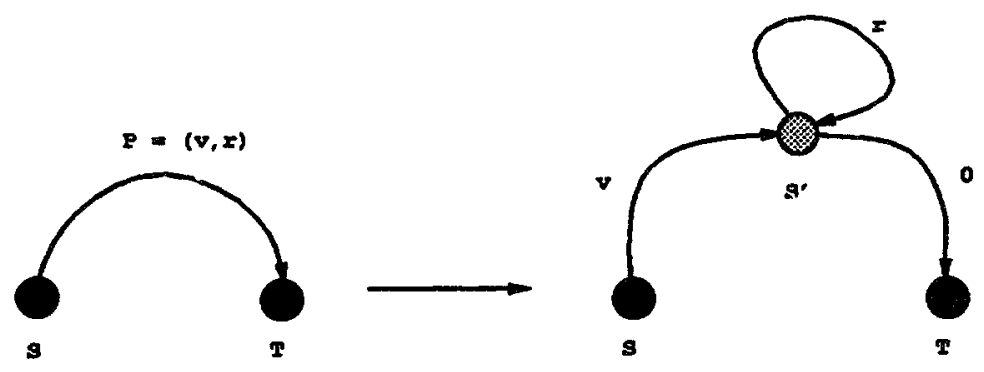

Fig. 12. Simulation of an edge labeled by a polyhedron with one vertex and one ray.

$r$ in Fig. 12). However, there are two fundamental differences between this framework and the classical framework of uniform loop nests:

- The uniform dependence vectors are not necessarily lexico-positive (for example, a ray can be equal to $(0,-1)$ ). Therefore, the scheduling problem is more difficult. However, it can be solved by techniques similar to those used to solve the problem of computability of systems of uniform recurrence equations. ${ }^{(7.22)}$

- The constraint imposed on a ray $r$ is weaker than the classical constraint: the constraint is indeed $X r \geqslant 0$ instead of $X r \geqslant 1$. This freedom must be taken into account in the parallelization algorithm.

\subsection{Dependence Abstractions}

For the sake of clarity, we restrict ourselves to the case of perfectly nested DO loops with affine loop bounds. Nonperfectly nested loops are considered in Section 7.3. This restriction allows us to identify, as usual, the iterations of $n$ nested loops ( $n$ is called the depth of the loop nest) with vectors in $\mathbb{Z}^{\prime \prime}$ (called the iteration vectors) contained in a finite convex polyhedron (called the iteration domain) defined by the loop bounds. The $i$ th component of an iteration vector is the value of the $i$ th loop counter in the nest, counting from the outermost to the innermost loop. In the sequential code, the iterations are therefore executed in the lexicographic order of their iteration vectors. In the next sections, we denote by $D$ the polyhedral iteration domain, by $I$ and $J$-dimensional iteration vectors in $\mathscr{D}$, and by $S_{i}$ the $i$ th statement in the loop nest. We write $I>, J$ if $I$ is lexicographically greater than $J$ and $I \geqslant, J$ if $I>, J$ or $I=J$.

Section 3.2.1 recalls the different concepts of dependence graphs: expanded dependence graphs (EDG), reduced dependence graphs (RDG), apparent dependence graphs (ADG) and the notion of distance sets. In 
Section 3.2.2, we formally define what we call polyhedral reduced dependence graphs (PRDG), i.e., reduced dependence graphs whose edges are labeled by polyhedra. Finally, in Section 3.2.3, we show how the model of PRDG generalizes classical dependence abstractions of distance sets.

\subsubsection{Dependence Graphs and Distance Sets}

Dependence relations between operations are defined by Bernstein's conditions. ${ }^{(26)}$ Briefly speaking, two operations are considered dependent if both operations access the same memory location and if at least one of the accesses is a write. The dependence is directed according to the sequential order, from the first executed operation to the last. Depending on the order of write(s) and/or read, the dependence corresponds to the so called flow dependence, anti dependence or output dependence. [In some cases, output and anti dependences can be removed by data renaming and data expansion. See for example Feautier. $\left.{ }^{(19)}\right]$ We write: $S_{i}(I) \Rightarrow S_{j}(J)$ if statement $S_{j}$ at iteration $J$ depends on statement $S_{i}$ at iteration $I$. The partial order defined by $\Rightarrow$ describes the expanded dependence graph (EDG). Note that $(J-I)$ is always lexicographically nonnegative when $S_{i}(I) \Rightarrow S_{j}(J)$.

In general, the EDG can not be computed at compile-time, either because some information is missing (such as the values of size parameters or even worse, precise memory accesses), or because generating the whole graph is too expensive. Instead, dependences are captured through a smaller (in general) cyclic directed graph, with s vertices, called the reduced dependence graph (RDG) (or statement level dependence graph). The RDG is a compression of the EDG. In the RDG, two statements $S_{i}$ and $S_{j}$ are said dependent (we write $S_{i} \rightarrow S_{j}$ ) if there exists at least one pair $(I, J)$ such that $S_{i}(I) \Rightarrow S_{j}(J)$. Furthermore, the dependence $S_{i} \stackrel{\leftrightarrow}{\longrightarrow} S_{i}$ is labeled by the set $\left\{(I, J) \in \mathscr{V}^{2} \mid S_{i}(I) \Rightarrow S_{j}(J)\right\}$, or by an approximation $D_{i}$, that contains this set. The precision and representation of this approximation makes the power of the dependence analysis. In other words, the RDG describes, in a condensed manner, an iteration level dependence graph, called (maximal) apparent dependence graph (ADG), that is a superset of the EDG. The ADG and the EDG have the same vertices, but the ADG has more edges, defined by:

$$
\begin{gathered}
\left(S_{i}, I\right) \Rightarrow\left(S_{i}, J\right) \text { (in the ADG) } \\
\Leftrightarrow \exists e=\left(S_{i}, S_{j}\right) \text { (in the RDG) such that }(I, J) \in D_{c}
\end{gathered}
$$

For a certain class of nested loops (see Feautier ${ }^{(1)}$ ), it is possible to express exactly this set of pairs $(I, J)$ : in this case, $I$ is given as an affine function $f_{i, j}$ of $J$ where $J$ varies in a polyhedron $: P_{i, j}$ :

$$
\left\{(I, J) \in \mathcal{X}^{2} \mid S_{i}(I) \Rightarrow S_{j}(J)\right\}=\left\{\left(f_{i, f}(J), J\right) \mid J \in \mathscr{P}_{i, j} \subset \mathscr{Z}\right\}
$$


In most dependence analysis algorithms however, rather than the set of pairs $(I, J)$, one computes the set $E_{i, j}$ of all possible values $(J-I), E_{i, j}$ is called the set of distance vectors, or distance set:

$$
E_{i, j}=\left\{(J-I) \mid S_{i}(I) \Rightarrow S_{j}(J)\right\}
$$

When exact dependence analysis is feasible, Eq. 3.1 shows that the set of distance vectors is the projection of the integer points of a polyhedron. This set can be approximated by its convex hull or by a more or less accurate description of a larger polyhedron (or a finite union of polyhedra). When the set of distance vectors is represented by a finite union, the corresponding dependence edge in the RDG is decomposed into multi-edges.

Note that the representation by distance vectors is not equivalent to the representation by pairs (as in Eq. 3.1), since the information concerning the location in the EDG of such a distance vector is lost. This may even be the cause of a loss of parallelism. However, this representation remains important, especially when exact dependence analysis is either too expensive or not feasible.

Classical representations of distance sets (by increasing precision) are:

- level of dependence, introduced for Allen and Kennedy's parallelizing algorithm. ${ }^{22.27)}$

- direction vector, introduced by Wolfe, ${ }^{(28.29)}$ and used in Wolf and Lam's algorithm. ${ }^{(4)}$

- dependence polyhedron (and dependence cone), introduced in Ref. 30 and used in Irigoin and Triolet's supernode partitioning algorithm. ${ }^{(3)}$ We refer to the PIPS ${ }^{(32)}$ software for more details on dependence polyhedra. Dependence cones are particular cases of dependence polyhedra.

We first define formally reduced dependence graphs whose edges are labeled by dependence polyhedra and we show the expressive power of this model.

\subsubsection{Polyhedral Reduced Dependence Graphs (PRDG)}

We first recall the mathematical definition of a polyhedron and how it can be decomposed into vertices, rays, and lines.

Definition 1. (Polyhedron, polytope) A set $P$ of vectors in $\mathbb{Q}^{\prime \prime}$ is called a (convex) polyhedron if there exists an integral matrix $A$ and an integral vector $b$ such that $P=\left\{x \mid x \in \mathbb{Q}^{\prime \prime}, A x \leqslant b\right\}$. A polytope is a bounded polyhedron.

$\times 2 \times 256.2$ 
A polyhedron can always be decomposed as the sum of a (convex) polytope and of a polyhedral cone (for more details see Ref. 33). A polytope is defined by its vertices, and any point of the polytope is a nonnegative barycentric combination of the polytope vertices. A polyhedral cone is finitely generated and can be defined by its rays and lines. Any point of a polyhedral cone is the sum of a nonnegative combination of its rays and of any combination of its lines. Therefore, a convex dependence polyhedron $P$ can be equivalently defined by a set of vertices (denoted by $\left.\left\{v_{1}, \ldots, v_{c o}\right\}\right)$, a set of rays (denoted by $\left.\left\{r_{1}, \ldots, r_{2},\right\}\right)$, and a set of lines (denoted by $\left.\left\{l_{1}, \ldots, l_{i}\right\}\right)$. Then, $P$ is the set of all vectors $p$ such that:

$$
p=\sum_{i=1}^{\prime \prime} \mu_{i} v_{i}+\sum_{i=1}^{p} v_{i} r_{i}+\sum_{i=1}^{j} \xi_{i} l_{i}
$$

with $\mu_{i} \in \mathbb{Q}^{+}, v_{i} \in \mathbb{Q}^{+}, \xi_{i} \in \mathbb{Q}$, and $\sum_{i=1}^{\prime \prime \prime} \mu_{i}=1$.

We now define what we call a polyhedral reduced dependence graph (or PRDG), i.e., a reduced dependence graph labeled by dependence polyhedra. Actually, we will be interested only in integral vectors that belong to the dependence polyhedra, since dependence distances are always integral vectors.

Definition 2. A polyhedral reduced dependence graph (PRDG) is a RDG, for which each edge $e: S_{i} \rightarrow S_{j}$ is labeled by a dependence polyhedron $P(e)$ that approximates the set of distance vectors: the associated ADG contains an edge from instance $I$ of node $S_{i}$ to instance $J$ of node $S_{j}$ if and only if $(J-I) \in P(e)$.

The notion of dependence polyhedron is very close to the notion of dependence convex hull..$^{(34)}$ Indeed, a dependence convex hull is a particular dependence polyhedron: the convex hull of the distance vectors. Here, we make no assumptions on the dependence polyhedra we handle or on the way they were computed.

In the rest of the paper, we explore this representation of dependences, where distance sets are approximated by polyhedra, specified by their vertices, rays, and lines. To avoid a possible confusion between the vertices of a dependence graph and the vertices of a dependence polyhedron, we call the first one nodes instead of vertices.

\subsubsection{Simulation of Classical Dependence Representations}

We now come back to more classical dependence abstractions: level of dependence and direction vector. We recall their definition and show that RDGs labeled by direction vectors or levels of dependence are actually particular cases of polyhedral reduced dependence graphs. 
3.2.3.1. Direction Vectors. When the set of distance vectors is a singleton, the dependence is said uniform and the only distance vector is called a uniform dependence vector. Otherwise, the set of distance vectors can still be represented by a n-dimensional vector (called the direction vector), whose components belong to $\mathbb{Z} \cup\{*\} \cup(\mathbb{Z} \times\{+,-\})$. Its $i$ th component is an approximation of the $i$ th components of all possible distance vectors: it is equal to $z+$ (resp. $z-$ ) if all $i$ th components are greater (resp. smaller) than or equal to $z$. It is equal to $*$ if the $i$ th component may take any value and to $z$ if the dependence is uniform in this dimension with unique value $z$. In general, $+($ resp. -$)$ is used as shorthand for $1+($ resp. $(-1)-)$.

We denote by $e_{i}$ the $i$ th canonical vector, i.e., the $n$-dimensional vector whose components are all zero except the $i$ th component which is equal to 1 . Then, a direction vector is nothing but an approximation by a polyhedron with a single vertex and whose rays and lines, if any, are canonical vectors. Indeed, consider an edge $e$ labeled by a direction vector $d$ and denote by $I^{+}, I^{-}$, and $I^{*}$ the sets of components of $d$ which are respectively equal to $z+$ (for some integer $z$ ), $z-$, and $*$. Finally, denote by $d$, the $n$-dimensional vector whose $i$ th component is equal to $z$ if the $i$ th component of $d$ is equal to $z, z+$, or $z-$, and to 0 otherwise. Then, by definition of the symbols,+- , and $*$, the direction vector $d$ represents exactly all $n$-dimensional vectors $p$ for which there exist integers $\left(v, v^{\prime}, \xi\right)$ in $\mathbb{N}^{\left|I^{+}\right|} \times \mathbb{N}^{\mid t^{-1}} \times \mathbb{Z}^{\left|I^{*}\right|}$ such that:

$$
p=d_{z}+\sum_{i \in I^{*}} v_{i} e_{i}-\sum_{i \in I^{*}} v_{i}^{\prime} e_{i}+\sum_{i \in I^{*}} \xi_{i} e_{i}
$$

In other words, the direction vector $d$ represents all integer points that belong to the polyhedron defined by the single vertex $d_{z}$, the rays $e_{i}$ for $i \in I^{+}$, the rays $-e_{i}$ for $i \in I$, and the lines $e_{i}$ for $i \in I^{*}$. For example, the direction vector $(2+, *,-, 3)$ defines the polyhedron with the vertex $(2,0,-1,3)$, the two rays $(1,0,0,0)$ and $(0,0,-1,0)$, and the line $(0,1,0,0)$.

3.2.3.2. Levels of Dependence. The representation by levels is the less accurate (but still useful on real applications) dependence abstraction. In a loop nest with $n$ nested loops, the set of distance vectors is approximated by an integer $l$, in $[1, \ldots, n] \cup\{\infty\}$, defined as the largest integer such that the $l-1$ first components of the distance vectors are zero. A dependence at level $l \leqslant n$ means that the dependence occurs at depth $l$ of the loop nest, i.e., at a given iteration of the $l-1$ outermost loops. In this case, one says that the dependence is a loop carried dependence at level $l$. If $l=\infty$, the dependence occurs inside the loop body, between two different statements, and is called a loop independent dependence. 
Consider an edge $e$ of level $l$. By definition of the level, the first non zero component of the distance vectors is the /th component and it can possibly take any positive integer value. Furthermore, we have no other knowledge on the remaining components. Therefore, an edge of level $l<\infty$ is equivalent to the direction vector $(0, \ldots, 0,1+, *, \ldots, *)$ (with $l-1$ leading zeros) and an edge of level $\infty$ corresponds to the zero dependence vector. As any direction vector admits an equivalent polyhedron, so does a representation by levels. For example, level 2 , in a three-dimensional loop nest, means direction vector $(0,1+, *)$ which corresponds to the polyhedron with one vertex $(0,1,0)$, one ray $(0,1,0)$, and one line $(0,0,1)$.

\section{OVERVIEW OF THE PARALLELIZATION ALGORITHM}

To help the reader follow, we first state the different steps of our parallelization algorithm, without entering neither into implementation details, nor into technical proofs. The detailed proofs of the correctness and optimality of our algorithm are delayed until Sections 5 and 6. An optimized version of the algorithm is presented in Section 7. Our parallelization algorithm consists of two main steps, a "uniformization" step presented in Section 4.1 and a "scheduling" step summarized in Section 4.2. We illustrate both steps with synthetic Example 5 .

Example 5. The code is given in Fig. 13. We assume that the edges of the reduced dependence graph (see Fig. 14) are labeled by direction vectors. This graph was built by the dependence analyzer Tiny. ${ }^{(35)}$

The reader can check that neither Allen and Kennedy's algorithm, nor Wolf and Lam's algorithm, is able to find the full parallelism for this code: the third statement seems to be purely sequential. However, the parallelism detection algorithm that we propose in the next sections is able to build the following multidimensional schedule: $(2 i+1,2 k)$ for the first statement,

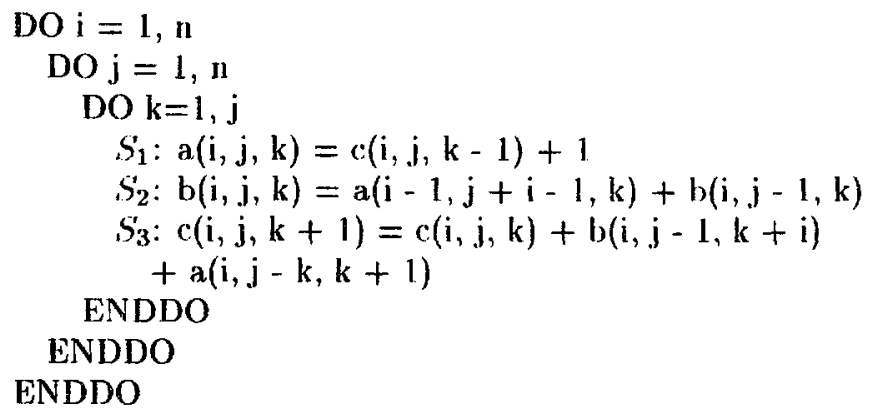

Fig. 13. Code for Example 5. 


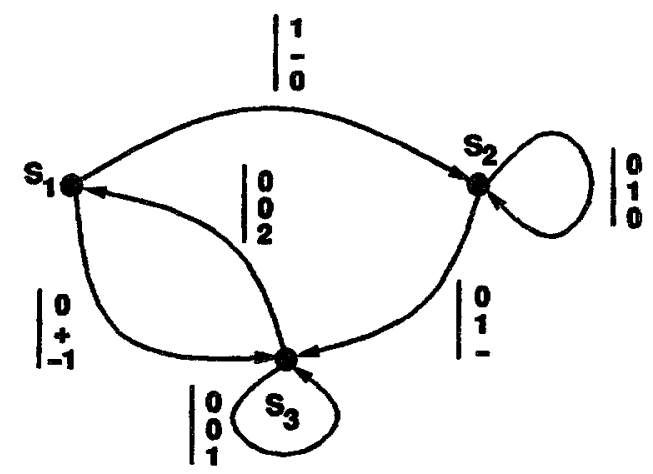

Fig. 14. RDG with direction vectors, Example 5.

$(2 i, j)$ for the second statement and $(2 i+1,2 k+3)$ for the third statement. This schedule corresponds to the code with explicit parallelism given Fig. 15 (but in which no effort, such as loop peeling, has been made so as to remove "if" tests). Thus, for each statement, one level of parallelism can be detected.

This code has been generated with the help of the procedure "codegen" of the Omega Calculator delivered with Petit. ${ }^{125)}$ The Omega Calculator, developed by Bill Pugh's team, is a framework to compute dependences, to check the validity of program transformations, and to transform programs, once the transformation is given. It does not propose

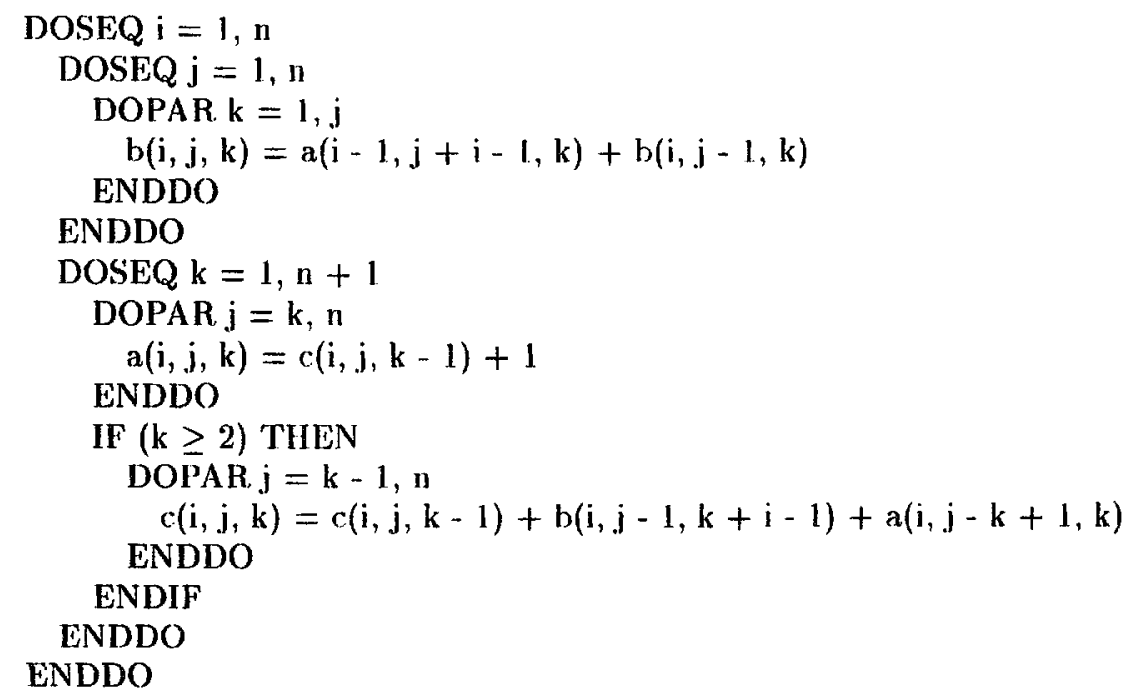

Fig. 15. Example 5, parallelized version. 
the transformations to be applied. We plan to integrate our algorithm in Petit as a transformation generator. We point out that the code proposed above is a "virtual" code in the sense that it only reveals hidden parallelism. We do not claim that it must be executed as such.

\subsection{Uniformization Step}

We first show how PRDGs (polyhedral reduced dependence graphs) can be captured into an equivalent (but simpler to manipulate) structure, the structure of uniform dependence graphs, i.e., graphs whose edges are labeled by constant dependence vectors. This uniformization scheme is achieved by the translation algorithm, given next.

To avoid possible confusions, the initial PRDG that describes the dependences in the code to be parallelized is called the original graph and denoted by $G_{o}=(V, E)$. The uniform RDG, equivalent to $G_{o}$, and built by the translation algorithm, is called the uniform graph or the translated of $G_{o}$ and is denoted by $G_{u}=(W, F)$.

The translation algorithm builds $G_{u}$ by scanning all edges of $G_{o}$. It starts from $G_{u}=(W, F)=(V, \varnothing)$, and, for each edge $e$ of $E$, it adds to $G_{u}$ new nodes and new edges depending on $P(e)$, the dependence polyhedron associated to $e$. We call virtual nodes the nodes that are created as opposed to actual nodes which correspond to nodes of $G_{0}$.

Let $e$ be an edge of $E$. We denote by $x_{c^{\prime}}$ and $y_{e^{\prime}}$, respectively the tail and head of $e$, i.e., the nodes that $e$ respectively leaves and enters: $x_{e} \stackrel{b}{\longrightarrow} y_{c}$. This definition is generalized to paths: the head (resp. tail) of a path is the head (resp. tail) of its last (resp. first) edge.

We follow the notations introduced in Section 3.2.2: we denote respectively by $\omega, \rho$, and $\lambda$ the number of vertices $v_{i}$, rays $r_{i}$, and lines $l_{i}$ of the polyhedron $P(e)$.

\subsubsection{Translation Algorithm}

- Let $W=V$ and $F=\varnothing$

- For $e: x_{c} \rightarrow y_{e} \in E$ do

(i) If $\rho=0, \lambda=0$, and $\omega=1$ (the polyhedron is a singleton, the dependence is uniform)

- Add to $F$ an edge of weight $v_{1}$ directed from $x_{c}$ to $y_{c}$.

(ii) If $\rho \neq 0$ or $\lambda \neq 0$ or $\omega>1$

- Add to $W$ a new virtual nod $n_{c}$,

- Add to $F \omega$ edges of weights $v_{1}, v_{2}, \ldots, v_{i,}$ directed from $x_{v^{\prime}}$, to $n_{v}$,

- Add to $F \rho$ self-loops around $n_{c}$ of weights $r_{1}, r_{2}, \ldots, r_{p}$, 
- Add to $F \lambda$ self-loops around $n_{c^{\prime}}$ of weights $l_{1}, l_{2}, \ldots, l_{\lambda}$,

- Add to $F \lambda$ self-loops around $n_{c}$ of weights $-l_{1},-l_{2}, \ldots,-l_{j}$,

- Add to $F$ a zero weight edge directed from $n_{c}$ to $y_{c}$.

Back to Example 5. The PRDG of Example 5 was depicted in Fig. 14. Figure 16 shows the uniform dependence graph associated to it. It has three new nodes (i.e., virtual nodes) that correspond to the symbol "+" and the two symbols ". -" in the initial direction vectors.

\subsection{Scheduling Step}

The scheduling step takes as input the translated dependence graph $G_{u}$ and builds a multi-dimensional schedule for each actual node, i.e., for each node of $G_{u}$ that corresponds to a node of $G_{o} . G_{u}$ is assumed to be strongly connected (otherwise, the algorithm is called for each strongly connected component of $G_{a r}$ ).

This is a recursive algorithm. Each step of the recursion builds a particular subgraph $G^{\prime}$ of the current graph $G$ being processed. Once $G^{\prime}$ is built, a set of linear constraints is derived and a valid schedule that satisfies all dependence edges not in $G^{\prime}$ can be computed. Then, the algorithm keeps working on the remaining edges, i.e. the edges of $G^{\prime}$ (more precisely $G^{\prime}$ and some additional edges, see later). Intuitively, the principle of the algorithm is to define a new order of computations (i.e., a loop transformation) such that as many dependence vectors as possible are carried by the outermost

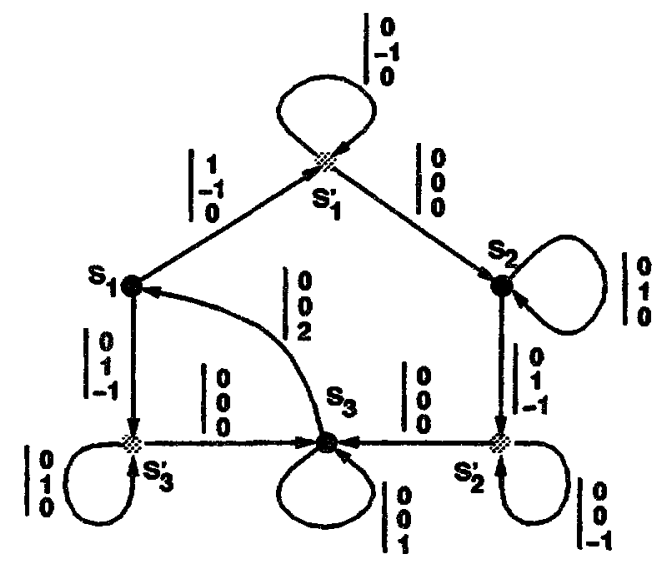

Fig. 16. Translated uniform redueed dependence graph for Example 5. 
loop after transformation, then as many remaining dependence vectors as possible are carried by the second outermost loop, and so on.

$G^{\prime}$ is defined as the subgraph of $G$ generated by all the edges of $G$ that belong to at least one multi-cycle of zero weight. A multi-cycle is a union of cycles, not necessarily connected, and the weight of a union of cycles is the sum of the weights of its constitutive cycles. $G^{\prime}$ is built by the resolution of a linear program (see Section 6.1 for details).

The scheduling step can be summarized by the recursive algorithm given later. The initial call is DARTE-VIVIEN $\left(G_{u}, 1\right)$. The algorithm builds, for each actual node $S$ of $G_{u}$ a sequence of vectors $X_{S}^{\mathrm{l}}, \ldots, X_{S}^{d_{S}}$ and a sequence of constants $\rho_{S}^{1}, \ldots, \rho_{S}^{d_{S}}$ that define a valid multi-dimensional schedule.

\subsubsection{DARTE-VIVIEN $(G, k) /^{*}$ builds the $k$ th component of the schedule *I}

(i) Build $G^{\prime}$ the subgraph of $G$ generated by all edges that belong to at least one zero weight multi-cycle of $G$.

(ii) Add in $G^{\prime}$ all edges from $x_{c}$ to $y_{c}$ and all self-loops on $y_{c}$ if $e=\left(x_{c}, y_{c}\right)$ is an edge already in $G^{\prime}$, from an actual node $x_{c}$ to a virtual node $y_{c}$.

(iii) Select a vector $X$ and, for each node $S$ in $G$, a constant $\rho_{S}$ such that:

$\left\{e=\left(x_{c}, y_{c}\right) \in G^{\prime}\right.$ or $x_{c}$ is a virtual node $\Rightarrow X w(e)+\rho_{y_{c}}-\rho_{v_{c}} \geqslant 0$

$\left\{e=\left(x_{i}, y_{c}\right) \notin G^{\prime}\right.$ and $x_{i}$ is an actual node $\Rightarrow X w(e)+\rho_{y_{r}}-\rho_{x_{e}} \geqslant 1$

For all actual nodes $S$ of $G$, let $\rho_{S}^{k}=\rho_{S}$ and $X_{S}^{k}=X$.

(iv) If $G^{\prime}$ is empty or has only virtual nodes, return.

(v) If $G^{\prime}$ is strongly connected and has at least one actual node, $G$ is not computable (and the initial PRDG $G_{o}$, is not consistent), return.

(vi) Otherwise, decompose $G^{\prime}$ into its strongly connected components $G_{i}$ and for each $G_{i}$, that has at least one actual node, call DARTE-VIVIEN $\left(G_{i}, k+1\right)$.

\section{Remarks.}

- We will see that Step (ii) is necessary only for general PRDGs: for example, it could be removed for RDGs labeled by direction vectors (see Section 6). In this case, the two steps, Step (i) and Step (iii), can be solved simultaneously by the resolution of a single linear program. 
- In Step (iii), we do not specify, on purpose, how the vector $X$ and the constants $\rho$ are selected, so as to allow various selection criteria. We refer to Section 7 for more details. For example, a maximal set of linearly independent vectors $X$ can be selected if the goal is to derive fully permutable loops (see Darte $e t a l^{(10)}$ ).

Back to Example 5. Consider the uniform dependence graph of Fig. 16. There are two elementary cycles of weights $(1,0,1)$ and $(0,1,1)$, and five self-loops of weights $(0,0,1),(0,0,-1),(0,1,0)$ (twice), and $(0,-1,0)$. Therefore, all edges (except the edges that only belong to the cycle of weight $(1,0,1))$ belong to a multi-cycle of zero weight. The subgraph $G^{\prime}$ is depicted in Fig. 17.

The constraints coming from edges in $G^{\prime}$ imply that $X=(x, y, z)$ must be orthogonal to the weight of all cycles of $G^{\prime}$. Therefore, $y=z=0$. Finally, considering the other constraints, we find the solution $X=(2,0,0)$, $\rho_{S_{1}}=\rho_{S_{3}}=1$ and $\rho_{S_{2}}=0$, for example if we minimize $x$.

In $G^{\prime}$, there remain four strongly connected components, and two of them are not considered since they only have virtual nodes. The two other components have no zero weight multi-cycles. The strongly connected component with the single node $S_{2}$ can be scheduled with the vector $X=(0,1,0)$, whereas studying the other strongly connected component leads, among other solutions, to $X=(0,0,2), \rho_{S_{1}}=0$ and $\rho_{S_{3}}=3$.

Finally, summarizing the results, we find, as claimed in the beginning of Section 4, the two-dimensional schedules: $(2 i, j)$ for $S_{2},(2 i+1,2 k)$ for $S_{1}$ and $(2 i+1,2 k+3)$ for $S_{3}$.

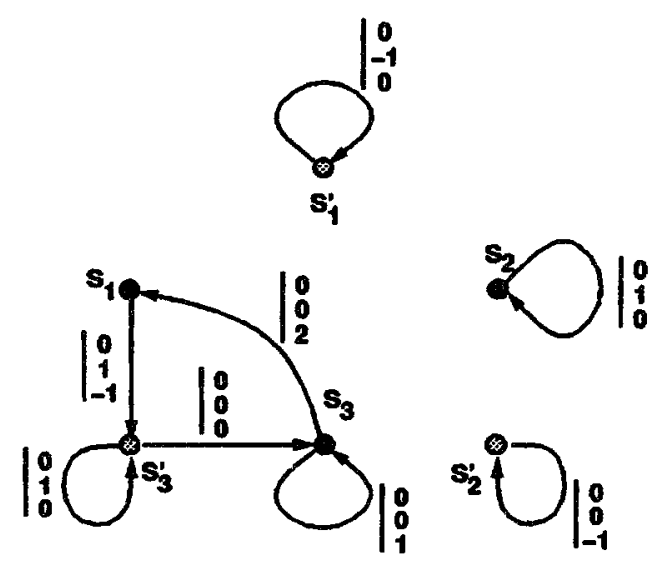

Fig. 17. Subgraph of zero weight multi-cycles for Example 5. 
We are now ready to explain in full details the different steps of this parallelization algorithm. It is based on scheduling techniques that we previously developed for systems of uniform recurrence equations. Therefore, we will frequently refer to Darte and Vivien. ${ }^{(22)}$

\section{COMPUTABILITY OF A PRDG}

In this section, we study the theoretical properties of PRDGs, in terms of computability, with the same spirit as in Karp et al. ${ }^{(7)}$ paper. Section 6 will show how computable PRDGs can be scheduled.

A dependence graph that defines exactly the dependences of a loop nest is always computable since, for example, the sequential ordering (the ordering of the initial non parallelized loop nest) defines a valid schedule. However, if dependences are given by an approximation, the dependence graph may describe a structure of computations that is not computable. For example, the PRDG of Fig. 18a is computable (even if the direction vector $(0, *)$ could be refined in the case of nested loops) whereas the PRDG of Fig. 18b is not computable, i.e., can not be scheduled. This comes from the fact that there is a dependence cycle of zero weight in the second PRDG, but not in the first one.

Therefore, before even thinking of parallelization, we need to check if a PRDG is computable or not. This section shows how it can be done. In Section 5.1, we explore the links between $G_{o}$ and its translated $G_{u}$. Following the results of this study, we are able to manipulate $G_{u}$, that has a more classical structure, instead of $G_{0}$. Indeed, $G_{\|}$is very similar to a reduced dependence graph associated to a system of uniform recurrence equations, i.e., a graph whose edges are labeled by integral vectors. We show how well-known results for systems of uniform recurrence equations can be adapted to $G_{u}$. Section 5.2 is devoted to the computability problem. Section 5.3 focuses on the length of the longest paths described by $G_{u}$. We

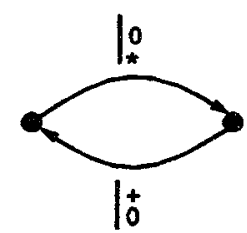

(a)

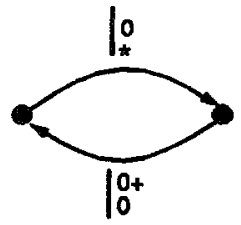

(b)

Fig. 18. Two different PRDGs: (a) computable; (b) not computable. 
give lower bounds on the length of these paths. These bounds are really important since lower bounds on the length of dependence paths are upper bounds on the parallelism in the dependence graphs (see Darte and Vivien '21" for a sharp definition of degree of parallelism).

From now on, we assume that the PRDG $G_{0}$ is strongly connected (and so is $G_{t}$ ). Otherwise, the results presented hereafter are true for each of the strongly connected components of $G_{a}$.

\subsection{Correspondence Between a PRDG $G_{0}$ and Its Translated $G_{\nu}$}

Recall that edges in $G_{c}$, are labeled, not by a particular weight (as in $G_{u}$ ), but by a polyhedron, i.e., a set of vectors. To avoid a possible confusion between edges labeled by a polyhedron and edges labeled by an integral vector, we call polyhedral edge $e$ an edge of $G_{o}$ labeled by a polyhedron $P(e)$, and dependence edge $e$ an edge of $G_{o}$ labeled by an integral vector $w(e)$ such that $w(e) \in P(e)$. In other words, a polyhedral edge e corresponds to as many dependence edges as there are integral vectors in $P(e)$ : a dependence edge is an instantiation of a polyhedral edge. We define a dependence path as a sequence of adjacent dependence edges. The weight of a dependence path is the sum of the weights of its edges.

We now show the links between paths in $G_{\imath}$ and dependence paths in $G_{11}$.

\subsubsection{From $G_{u}$ to $G_{o}$}

Note first the particular structure of $G_{u}$. There is no edge between distinct virtual nodes. Thus, any path between two distinct virtual nodes has to pass through an actual node. Furthermore, any path between two actual nodes whose intermediate nodes are all virtual nodes, visits only one virtual node, possibly many times: we call such a path a basic path. The link between basic paths in $G_{u}$ and edges in $G_{0}$ is straightforward, given by the following lemma and corollary:

Lemma 1. A basic path $\Pi_{u}$ of $G_{u}$ corresponds to a unique polyhedral edge $e$ of $G_{a}$ and the total weight of $\Pi_{u}$ is a vector that belongs to the polyhedron $P(e)$ associated to $e$.

Proof. The proof is straightforward, by construction of $G_{u}$. A detailed proof is given in Darte and Vivien. ${ }^{36)}$

Corollary 1. A path $\Pi_{\|}$of $G_{u}$, from an actual node to an actual node, defines an equivalent dependence path $\Pi_{0}$ in $G_{a}$ : each basic sub-path 
of $\Pi_{u}$ corresponds exactly to a polyhedral edge $e$ of $G_{o}$ whose dependence polyhedron contains the weight of the basic sub-path.

\subsubsection{From $G_{o}$ to $G_{u}$}

Lemma 1 (and a fortiori Corollary 1) is in general not a strict equivalence: it is not always possible to build an equivalent basic path in $G_{t \prime}$ for any dependence edge of $G_{o}$, since an integral vector in $P(e)$ may be a rational (but not integral) linear combination of vertices, rays and/or lines of $P(e)$. However, when the dependence path is a cycle, there is still a correspondence as stated in Lemma 2 . In the following, if $\mathscr{C}$ is a cycle, $m \mathscr{C}$ denotes the cycle formed by $m$ times the cycle $\mathscr{C}$.

Lemma 2. Let $\mathscr{C}$ be a dependence cycle of $G_{o}$, i.e., a cycle of dependence edges. Then, for some integer $m$, the cycle $m \mathscr{C}$ is equivalent to a cycle $\mathscr{C}_{u}$ in $G_{u}$ of same structure and same weight.

Proof. The proof is not difficult. One has just to decompose each polyhedral edge on the vertices, rays and lines of the corresponding polyhedron. If some components are not integral, we multiply (i.e., we use several times the cycle) all the components by a suitably large integer so that they all become integral. Details can be found in Darte and Vivien. ${ }^{(36)}$

\subsubsection{Computability Condition}

In the case of bounded iteration domains, a reduced dependence graph is computable if and only if the apparent dependence graph it describes is acyclic, i.e., if there is no dependence path from an instance of a statement to the same instance of the same statement. In other words, a PRDG $G_{o}$ is computable if and only if it has no dependence cycle of zero weight. According to Corollary 1 and Lemma 2 , the computability of $G_{o}$ is translated into $G_{u}$ as follows:

Theorem 1. $G_{o}$ is computable if and only if $G_{u}$ contains no zero weight cycle with at least one actual node.

\subsection{Computability of the Translated Graph $G_{u}$}

In the previous section, we moved the computability problem from $G_{o}$ to $G_{u}$. Now, checking the existence of zero weight cycles in $G_{u}$ is simpler than in $G_{o}$ since $G_{u}$ is nothing but the reduced dependence graph of a system of uniform recurrence equations, except that some nodes are called actual and some other virtual. We can thus apply all techniques developed 
for system of uniform recurrence equations (see Refs. 7 and 22), with a slight modification so as to take into account the fact that some nodes in $G_{u}$ are virtual nodes and exist only to simulate dependences in $G_{w}$.

Many algorithms that check the computability of systems of uniform recurrence equations have been proposed, ${ }^{(7.22 .37 .39)}$ the first one being Karp et al. decomposition. ${ }^{(7)}$ However, we suggest the reader to refer to the algorithm formulation and proofs proposed by Darte and Vivien, ${ }^{221}$ because they have been developed with the scheduling problem (more than with the computability problem) in mind, which is more interesting from the point of view of parallelization.

The basic algorithm looks for zero weight cycles in a uniform reduced dependence graph $G$. It is a recursive algorithm, based on the search for zero weight multi-cycles (i.e., union of cycles, not necessarily connected). However, in our case (cf. Theorem 1), we are not interested in all zero weight cycles, but only in zero weight cycles which contain an actual node. We have thus to refine the decomposition algorithm. This leads to the following algorithm which enables us to determine the computability of $G_{o}$ via $G_{u}$. The only modification we make compared to Karp et al. decomposition $^{(7)}$ is to not consider subgraphs of $G_{u}$ that have only virtual vertices. Therefore, the correctness of this new algorithm is a direct consequence of the correctness of Karp et al. decomposition.

\subsubsection{Decomposition Algorithm: $D A$}

$\wedge$ denotes the logical AND.

\subsubsection{Boolean $D A(G)$}

(i) Build $G^{\prime}$ the subgraph of $G$ generated by all the edges that belong to a zero weight multi-cycle of $G$.

(ii) Compute the strongly connected components of $G^{\prime}$ and let $G_{1}^{\prime}$, $G_{2}^{\prime}, \ldots, G_{s}^{\prime}$ be the $s$ components that have at least one actual node.

- If $G^{\prime}$ is empty or has only virtual nodes, return TRUE.

- If $G^{\prime}$ is strongly connected and has at least one actual node, return FALSE.

- Otherwise, return $\wedge_{i=1}^{s} \operatorname{DA}\left(G_{i}^{\prime}\right)$.

Then, $G_{u}$ has no cycle of zero weight containing an actual node if and only if DA $\left(G_{u}\right)=$ TRUE. Therefore, a PRDG $G_{w}$ is computable if and only if $\mathrm{DA}\left(G_{u}\right)=$ TRUE. 
Definition 3. For a graph $G_{0}$, we define the depth of $G_{o}$ (denoted by $d\left(G_{o}\right)$ or simply $d$ ) as the maximal number of recursive calls generated by the initial call $\operatorname{DA}\left(G_{u}\right)$ (counting the first one), except if $G_{v}$ is acyclic, in which case we let $d\left(G_{n}\right)=0$.

For systems of uniform recurrence equations, the depth of the decomposition algorithm is a measure of the degree of parallelism described by its dependence graph. ${ }^{(16)}$ We will see that this result still holds for PRDGs and the depth $d$ defined for algorithm DA.

\subsubsection{Dependence Polyhedra Considered to be Atomic: Algorithm DA*}

For technical reasons that will become clear in the scheduling section (Section 6), we need to slightly modify the decomposition algorithm DA.

Remember how $G_{u}$ was built (refer to the translation algorithm in Section 4.1). We want to keep together, during the decomposition algorithm, all edges that come from the translation of the same dependence polyhedron $P(e)$, as soon as one of the vertices of $P(e)$ is kept in $G^{\prime}$. For that, we add in $G^{\prime}$ all edges from $x_{c}$ to $y_{e}$ and all self-loops on $y_{e}$ if $e=\left(x_{e}, y_{c}\right)$ is an edge already in $G^{\prime}$, from an actual node $x_{e}$ to a virtual node $y_{c}$. This leads to the following algorithm:

\subsubsection{Boolean $D A *(G)$}

(i) Build $G^{\prime}$ the subgraph of $G$ generated by all the edges that belong to at least one zero weight multi-cycle of $G$.

(ii) Add in $G^{\prime}$, all edges from $x_{c}$, to $y_{c}$ and all self-loops on $y_{c}$ if $e=\left(x_{c}, y_{c}\right)$ is an edge already in $G^{\prime}$, from an actual node $x_{c}$ to a virtual node $y_{a}$.

(iii) Compute the strongly connected components of $G^{\prime}$ and let $G_{1}^{\prime}, G_{2}^{\prime}, \ldots, G_{s}^{\prime}$ te the $s$ components that have at least one actual node.

- If $G^{\prime}$ is empty or has only virtual nodes, return TRUE.

- If $G^{\prime}$ is strongly connected and has at least one actual node, return FALSE.

- Otherwise, return $\bigwedge_{i=1}^{*} \mathrm{DA}^{*}\left(G_{i}^{\prime}\right)$.

Note that the edges we add to $G^{\prime}$ do not change the structure of strongly connected components of $G^{\prime}$, since we add edges only between nodes that already belong to the same strongly connected component of $G^{\prime}$. Furthermore, in each strongly connected component, the structure of zero weight multi-cycles is also unchanged since we add only edges that do not belong to a zero weight multi-cycle. Therefore, both algorithms $\mathrm{DA}^{*}$ and DA have exactly the same behavior (in terms of nodes). 


\subsection{Longest Dependence Paths in $G_{0}$}

Once we know whether a polyhedral reduced dependence graph is computable, it is interesting to give an estimate on the length of the longest path in its apparent dependence graph. This length gives a lower bound on the sequentiality described by the PRDG, and thus, gives an upper bound on the parallelism it contains.

In Darte et al. ${ }^{(17)}$ it is shown that, for a single uniform recurrence equation (i.e., a uniform reduced dependence graph with a single node), this length is equivalent to the latency of the optimal linear schedule, for full dimensional polyhedral iteration domains whose size tends to infinity. Remember that the latency of a linear schedule is nothing but the number of sequential iterations. To say it briefly, on domains of size parameterized by $N$, the latency of the optimal linear schedule is equivalent to $\lambda N$ for some constant $\lambda$ and so does the length of the longest dependence path. Both are in $N$ and the multiplicative constants are the same. This result has been extended in Ref. 18, for strongly connected uniform dependence graphs, if a "shifted linear" schedule exists.

In our case, which is similar to the case of general uniform dependence graphs, one-dimensional affine schedules may not exist and the length of the longest path is not necessarily $O(N)$ anymore, it can be equivalent to $k N^{\prime \prime}$ for some constants $k$ and $p$. We will not try to be precise on the multiplicative constant $k$, we will just focus on $p$ the power of $N$.

Actually, for a system of uniform recurrence equations, it has been shown that this power is equal to the depth of Karp et al. decomposition ${ }^{(7)}$ as recalled by the following theorem (Theorem 2). Indeed, if $d$ is the depth of $G$ for Karp et al. decomposition, there exists a path in the apparent dependence graph of length $\Omega\left(N^{d}\right)$. A complete proof of this result can be found in Darte and Vivien. ${ }^{(4))}$ The iteration domain is supposed to contain (resp. to be contained in) a full dimensional cube of size $\Omega(N)$ (resp. $O(N))$. Actually, we have the following stronger result:

Theorem 2. Let $G$ be the dependence graph of a system of uniform recurrence equations. For each node $S$ of $G$, let $d_{S}$ be the depth where $S$ is removed, in Karp et al. decomposition. (7) Then, for each strongly connected component $G^{i}$ of $G$, there exists a path in the apparent dependence graph of $G$ whose projection in $G$ is a cycle that visits $\Omega\left(N^{d s}\right)$ times each node $S$ of $G^{i}$.

A similar theorem can be stated for PRDGs:

Theorem 3. Let $G_{a}$ be a PRDG, and $G_{u}$ the corresponding translated graph. For each node $S$ of $G_{t}$, let $d_{S}$ be the depth where the node $S$ 
is removed, in the decomposition algorithm DA (or equivalently DA*) applied to $G_{u}$. Then, for each strongly connected component $G_{o}^{i}$ of $G_{o}$, there exists a path in the apparent dependence graph of $G_{o}$ whose projection in $G_{o}$, is a cycle that visits $\Omega\left(N^{d} s_{s}\right)$ times each node of $G_{o}^{i}$.

Proof. Note first that for each actual node of $G_{u}$ the depth for Karp et al. decomposition ${ }^{(7)}$ (in which actual nodes and virtual nodes are not distinguished) and the depth for Algorithm DA are equal. We can thus apply Theorem 2 which gives, for each strongly connected component $G_{u}^{i}$ of $G_{u}$, a path in the apparent dependence graph of $G_{u}$, whose projection in $G_{u}$ visits $\Omega\left(N^{d s}\right)$ times each node $S$ of $G_{u}^{i}$. Furthermore, the projection in $G_{u}$ is a cycle with at least one actual node. We then use Corollary 1 to obtain an equivalent path in $G_{o}$. Lemma 1 ensures that the number of occurrences of an actual node in both paths are equal.

\section{SCHEDULING A PRDG}

In this section, we detail the scheduling algorithm for PRDGs introduced in Section 4.2. In particular, we show how to find the vector $X$ and the constants $\rho_{s}$. We also prove that our algorithm is able to find all the parallelism contained in a PRDG. In Section 6.1, we recall how $G^{\prime}$ can be computed and the link with "shifted-linear" schedules. In Section 6.2, we show that the scheduling algorithm is correct, i.e., that it produces a schedule that respect all dependences. Finally, in Section 6.3, we show the optimality of the scheduling algorithm.

\subsection{Construction of $G^{\prime}$ and Related Properties}

In this section, we recall how to build $G^{\prime}$, the subgraph of zero weight multi-cycles. We also recall why it is closely related to the construction of separating hyperplanes and, thus, to the construction of multidimensional schedules.

Step (i) of the decomposition algorithm (DA or DA*), devoted to the construction of $G^{\prime}$, can be implemented by the resolution of a single linear program. Indeed, it has been shown (see Darte and Vivien ${ }^{(22)}$ ) that the edges of $G^{\prime}$ are exactly the edges $e$ for which $v_{c}=0$ in any optimal solution of the following linear program:

$$
\min \left\{\sum_{c} v_{c} \mid q \geqslant 0, v \geqslant 0, w \geqslant 0, q+v=w+1, C q=0, W q=0\right\}
$$


where $C$ is the connection matrix of $G$ (with as many rows as nodes in $G$, and as many columns as edges in $G$ ) and $W$ the dependence matrix (i.e., whose columns are the weights of edges of $G$ ). This linear program (Program 6.1) is linked to the computability problem, whereas its dual (Program 6.2) is linked to the scheduling problem:

$$
\max \left\{\sum_{\iota^{\prime}} z_{c} \mid 0 \leqslant z \leqslant 1, X w(e)+\rho_{y_{e}}-\rho_{x_{r}} \geqslant z_{c^{\prime}}\right\}
$$

The relation between both programs is the following. Consider the set $\sum$ defined by:

$$
\Sigma=\left\{(X, \rho) \mid X w(e)+\rho_{x_{r}}-\rho_{x_{e}} \geqslant 0 \text { for all } e \in G\right\}
$$

The set $\sum$ is fundamental for deriving fully permutable loops nests (see Darte et al.. ${ }^{(10)}$ Edges of $G^{\prime}$ are characterized by the following lemma:

\section{Lemma 3.}

- $e \in G^{\prime} \Leftrightarrow \forall(X, \rho) \in \sum, X w(e)+\rho_{y_{c}}-\rho_{x_{c}}=0$.

- $\exists(X, \rho) \in \sum$ such that $\forall e \notin G^{\prime}, X w(e)+\rho_{v_{c}}-\rho_{x_{r}} \geqslant 1$.

Lemma 3 shows that considering the dual provides separating hyperplanes, which are strictly separating hyperplanes for the edges not in $G^{\prime}$ and weakly separating hyperplanes for edges in $G^{\prime}$. Furthermore, any optimal solution of the dual program provides a separating hyperplane that is a strictly separating hyperplane simultaneously for all edges of $G^{\prime}$. In particular if $G^{\prime}$ is empty, Lemma 3 enables to build a "shifted linear" schedule.

\subsection{Correctness of the Scheduling Algorithm}

We are now ready to prove that the scheduling algorithm proposed in Section 4.2 is correct.

Note first that the skeleton of Algorithm DARTE-VIVIEN is nothing but Algorithm DA*, whose correctness has already been studied in Section 5.2.2. Therefore, it remains to prove two things:

- that Step (iii) is feasible, i.e., that it is possible to define a vector $X$ and some constants $\rho_{S}$ satisfying the desired inequalities. 
- that, as claimed in Section 4.2, the sequence of vectors $X_{S}^{1}, \ldots, X_{S}^{d_{S}}$ and the sequence of constants $\rho_{S}^{t}, \ldots, \rho_{S}^{d_{s}}$ define a valid multi-dimensional schedule.

The first point is clear. Indeed, from Lemma 3, we know that there exists a vector $X$ and some constants $\rho_{S}$ (for example, any optimal solution of the dual program (Program 6.2)) such that:

$$
\left\{\begin{array}{lll}
X w(e)+\rho_{v_{c}}-\rho_{x_{c}}=0 & \text { for all } & e \in G^{\prime} \\
X w(e)+\rho_{v_{e}}-\rho_{v_{c}} \geqslant 1 & \text { for all } & e \notin G^{\prime}
\end{array}\right.
$$

Therefore, the constraints of Step (iii) can be satisfied since they are weaker (some inequalities $\geqslant 1$ are relaxed to inequalities $\geqslant 0$ ).

For making the proof of the second point simpler, we complete each sequence of vectors $X_{S}^{i}$ with null vectors and each sequence of constants $\rho_{S}^{i}$ with null constants so as to define sequences of same length $d$ (remember that $d$ is the depth of the decomposition algorithm DA on $G_{u}$ ).

Lemma 4. If $G_{c}=(V, E)$ is computable, the multi-dimensional scheduling function $T$ :

$$
\begin{aligned}
& V \times \mathscr{D} \longrightarrow \mathbb{Z}^{\prime \prime} \\
& (S, I) \longrightarrow\left(\ldots,\left\lfloor X_{S}^{i} I+\rho_{S}^{i}\right\lrcorner, \ldots\right)
\end{aligned}
$$

defines a valid multi-dimensional schedule for $G_{n}$.

Proof. A multi-dimensional scheduling function defines, for each statement $S$ (i.e., each node of $G_{c}$ ) and for each iteration vector $I \in \mathscr{D}$, a multi-dimensional vector $T(S, I)$. Then, computations are scheduled by lexicographic order of the $T(S, I)$. Therefore, in order to prove that $T$ is a valid schedule, we need to prove that all dependence distances remain lexico-positive, i.e., that for all edges $e=\left(x_{c}, y_{c}\right)$ of $G_{o}$, for any weight $w(e)$ in $P(e)$, and for all point $I$ of the domain $D$, we have $T\left(y_{c}, I\right)>$, $T\left(x_{e}, I-w(e)\right)$ where $<$, denotes the strict lexicographic order (see Section 3.2). Actually, we prove a bit stronger property: there exists an integer $k \in[1, d]$ such that

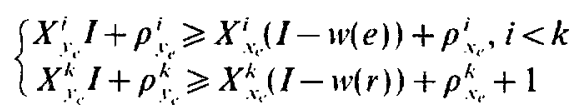

which will imply the desired property $T\left(y_{\iota}, I\right)>, T\left(x_{e}, I-w(e)\right)$. 
Remember that all possible values of $w(e)$ are given by Eq. (3.2): $w(e)=\sum_{j=1}^{j=i} \mu_{j} v_{j}+\sum_{j=1}^{j=p} v_{j} r_{j}+\sum_{j=1}^{j=i} \xi_{j} l_{j}$ with $\mu_{i} \in \mathbb{Q}^{+}, v_{i} \in \mathbb{Q}^{+}, \xi_{i} \in \mathbb{Q}$ and $\sum_{j=1}^{j=n^{\prime \prime}} \mu_{j}=1$. We built $G_{u}$ the following way. For each edge $e=\left(x_{i}, y_{e}\right)$, we introduced a new node $n_{c}$. In $G_{u}$, all edges from $x_{c}$, to $n_{c}$ correspond to vertices of $P(e)$, all self-loops on $n_{c}$ correspond to rays or lines, and there is a zero weight edge from $n_{c}$ to $y_{c}$.

If $G_{o}$ is computable, all recursive calls in the decomposition algorithm DA end because the current $G^{\prime}$ is empty or has only virtual nodes. Thus, each edge from $x_{c}$, to $n_{c}$ is removed from the graph (i.e., do not belong to the current $G^{\prime}$ ) at some level of the decomposition. We denote by $k$ the level where all edges that correspond to a vertex of $P(e)$ (i.e., edges from $x_{\varepsilon}$ to $n_{\varepsilon}$ ) have been finally removed. Because of Step (ii) of algorithm DA* (equivalent to Step (ii) of the scheduling algorithm), all edges that correspond to $P(e)$ are actually kept together in the graph, until level $k$. More precisely, they are kept together in the same strongly connected component until level $k$, as well as the zero weight edge from $n_{c}$, to $y_{c}$.

Therefore, the sequences of vectors $X_{v i}^{i}, X_{x_{i}}^{i}$ and $X_{n_{i}}^{i}$, are equal until level $k$ : we can thus skip the subscript and we simply write $X^{i}$. The system 6 is reduced to:

$$
\left\{\begin{array}{l}
X^{i} w(e)+\rho_{s}^{i}-\rho_{v_{c}}^{i} \geqslant 0 \\
X^{k} w(e)+\rho_{s}^{k}-\rho_{x_{c}}^{k} \geqslant 1
\end{array} \quad \text { for all } i, 1 \leqslant i \leqslant k-1\right.
$$

Now, refer to Step (iii) of the scheduling algorithm. Until level $k$, we have:

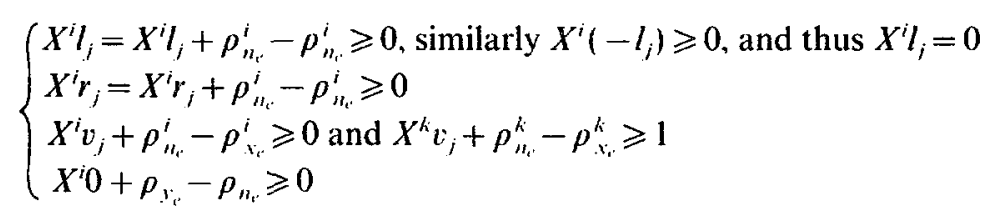

Thus, with the definition of $w(e)$, we get the desired result: all constraints of System 6.4 are satisfied and are obtained by summing these inequalities in a suitable way.

Remark. The modification we made to keep together all edges corresponding to the polyhedron $P(e)$ becomes clear. It enables to ensure that 
all constraints of System 6.3 are satisfied, even if $w(e)$ is a rational combinations of vertices, rays, and lines of $P(e)$. Actually, if for all integral vectors $w(e) \in P(e)$, the decomposition of Eq. (3.2) is a decomposition with integral (instead of rationals) coefficients - in this case, one says that $P(e)$ is decomposed on a Hilbert basis, see Schrijver ${ }^{133}$ - then, the algorithm can be refined: edges $f$ in $G_{u}$ corresponding to $P(e)$ and for which $X w(f)+$ $\rho_{1}-\rho_{x_{t}} \geqslant 1$ in Step (iii) do not have to be kept in $G^{\prime}$. This enables to relax constraints for the next levels. This is the case for example for direction vectors. The decomposition proposed in Section 3.2.3 is indeed a decomposition on a Hilbert basis.

\subsection{Optimality of the Multi-Dimensional Schedule}

Now that we have a multi-dimensional schedule $T$, we prove its optimality in terms of degree of parallelism. We want to show that for each statement $S$ (i.e., for each node of $G_{o}$ ), the number of instances of $S$ that are sequentialized by $T$ is of the same order as the number of instances of $S$ that are inherently sequentialized by the dependences.

The latency of $T$ is the number of sequential steps induced by the order defined by $T$. In order to be more precise, we define for a statement $S$, the $S$-latency of $T$ as the number of instances of $S$ sequentialized by $T$. If the iteration domain $\mathscr{D}$ is contained in a $n$-dimensional cube of size $O(N)$, then the $S$-latency of $T$ is $O\left(N^{d} s\right)$ since instances of $S$ are scheduled by a $d_{S}$-dimensional schedule. Similarly the latency of $T$ is $O\left(N^{d}\right)$. We define the $S$-length of a dependence path $\mathscr{P}$ in the apparent dependence graph associated to $G_{o}$ as the number of vertices in $\mathscr{P}$ that correspond to instances of $S$. Therefore, the $S$-length of a dependence path $\mathscr{P}$ is the number of instances of $S$ in $\mathscr{P}$ that are inherently sequentialized by the dependences. Theorem 3 shows that there is a dependence path whose $S$-length is $\Omega\left(N^{d} s\right)$ if the iteration domain $\mathscr{D}$ contains a $n$-dimensional cube of size $\Omega(N)$. This proves the following result:

Theorem 4. The scheduling algorithm is nearby optimal: if the iteration domain contains (resp. is contained in) a full dimensional cube of size $\Omega(N)$ (resp. $O(N)$ ), and if $d$ is the depth of the decomposition algorithm, then, the latency of the schedule is $O\left(N^{d}\right)$ and the length of the longest dependence path is $\Omega\left(N^{d}\right)$. More precisely, after code generation, each statement $S$ is surrounded by exactly $d_{S}$ sequential loops and these loops are inherently sequential. 


\section{IMPLEMENTATION, EXAMPLES, AND EXTENSIONS}

\subsection{Implementation Strategies}

The most time consuming steps of the scheduling algorithm presented in Section 4.2 are the resolutions of the different linear programs of Steps (i) and (iii). In order to speed up the algorithm, we replace the linear programs previously described by some equivalent linear programs with fewer variables and fewer equations.

Consider the constraints of Step (iii). It turns out that they are equivalent to the constraints $X w(C) \geqslant l(C)$ for all elementary cycles $C$ where $l(C)$ denotes the number of edges $e=\left(x_{c}, y_{c}\right)$ of $C$ that do not belong to $G^{\prime}$ and for which $x_{c}$ is an actual node. Furthermore, once the constraints on cycles are satisfied, the different constants $\rho$ can be computed by a technique similar to the Bellman-Ford algorithm, less expensive than the resolution of a linear program.

This remark would enable to reduce the number of variables in the linear programs. Unfortunately, it would increase the number of constraints, since the number of elementary cycles in the graph may be exponential in the number of edges. Therefore, computing the weight of all cycles in the PRDG may not be always reasonable. To avoid this problem, we use a basis of cycles, instead of considering all cycles. This technique enables to reduce both the number of constraints and the number of variables in the linear program of Step (iii). Later, we only give an idea of this technique. Details can be found in Darte and Vivien. ${ }^{(36)}$

Definition 4. A basis of cycles $\mathscr{B} \mathscr{C}$ of $G$ is a family of independent cycles $^{5} C_{1}, \ldots, C_{m}$ such that for each cycle $C$ in $G$, there exists some rationals $\lambda_{1}, \ldots, \lambda_{m}$ such that: $C=\sum_{i=1}^{m} \lambda_{i} C_{i}$.

We refer to Gondran and Minoux ${ }^{411}$ for a more precise definition of basis of cycles and of cyclomatic number.

\subsubsection{Computation of the Vector $X$}

For each edge $e=\left(x_{c}, y_{c}\right)$ of $G$, we define an integer $\delta_{c}$, equal to 0 if $e$ is in $G^{\prime}$ or if $x_{c}$ is a virtual statement, and equal to 1 otherwise, i.e., if $e$

\footnotetext{
"Actually, in such "cycles," edges can be traversed backwards. Except in this definition, all cycles used in the rest of the paper are "true" cycles, i.e., cycles for which edges are always traversed forwards.
} 
is not in $G^{\prime}$ and if $x_{e}$ is an actual statement. Then, the constraints to solve are:

$$
\{x \geqslant 0, X \mathscr{W}=(x+\delta) Q\}
$$

Actually, this system is only a set of constraints, with no objective function. This allows us to define many objective functions so as to optimize the choice of $X$. Currently, we minimize its norm $\sum_{i=1}^{\prime \prime}\left|X_{i}\right|$. This is done by the following linear program:

$$
\min \left\{\sum_{i=1}^{n} Y_{j} \mid x \geqslant 0, X \mathscr{W}^{-}=(x+\delta) Q, X \leqslant Y,-X \leqslant Y\right\}
$$

In the following, we assume that we have a solution $(X, x)$ of the linear program 7.1.

\subsubsection{Computation of the Constants $\rho$}

To compute the constants $\rho$, we use the Bellman-Ford algorithm ${ }^{(42)}$ which solves the single-source shortest-path problem. The Bellman-Ford algorithm determines whether or not there is a negative-weight cycle that is reachable from the source. If there is no such cycle, the algorithm produces the shortest paths and their weights. It runs in time $O(|V \| E|)$.

We build a copy $H$ of the graph $G$. For each edge $e$ of $G$, we replace its distance vector weight $w(e)$ by the induced delay $w^{\prime}(e)$ defined by $w^{\prime}(e)=X w(e)-\delta_{c}$. As we want to use the Bellman-Ford algorithm on $H$, we need to prove that $I$ contains no negative-weight (true) cycles.

Lemma 5. The weight of any (true) cycle $\mathscr{C}$ in $H$ is nonnegative.

Proof. Consider a true cycle $\mathscr{C}$ in $H: \mathscr{C} \geqslant 0$. Decompose $\mathscr{C}$ on the basis of cycle $\mathscr{B} \mathscr{C}: \mathscr{C}=Q \lambda$ for some vector $\lambda$. The weight of $\mathscr{C}$ in $H$ is:

$$
w^{\prime}(\mathscr{C})=w^{\prime}(Q \lambda)=(X \mathscr{W}-\delta Q) \lambda=x Q \lambda=x \mathscr{C}
$$

Since both vectors $x$ and $\mathscr{C}$ are nonnegative by definition, $x \mathscr{C}$ is nonnegative.

Then, we use the Bellman-Ford algorithm on $H$ to produce the shortest paths and their weights. We denote by $\rho_{S}$ the opposite of the weight of the shortest path to the node $S$. 


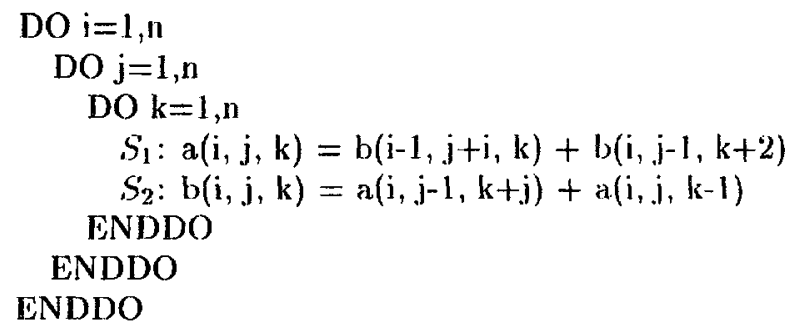

Fig. 19. Code of Example 6.

Lemma 6. The vector $X$ and the constants $\rho_{S}$ satisfy the conditions of Step (iii) of the scheduling algorithm.

Proof. Let $e=\left(x_{c}, y_{c}\right)$ be an edge of $G$. The delay induced on $e$ by $\left(X, p_{c}\right)$ is equal to $X w(e)+\rho_{x,}-\rho_{x,}$. By definition of a shortest path, we have:

$$
\left(-\rho_{y_{c}}\right) \leqslant\left(-\rho_{v_{c}}\right)+w^{\prime}(\mathcal{e}) \Leftrightarrow X w(e)+\rho_{v_{c}}-\rho_{x_{c}} \geqslant \delta_{c}
$$

which is the desired inequality.

This two steps strategy, computing $X$ first by a linear programming approach, and then the constants $\rho$ by a graph-based technique, is the way we implemented the scheduling algorithm. It is not clear from a practical point of view if it really speeds up the linear program resolutions. However, its main interest is that it enables to guarantee that the constants $\rho$ are simple if the vector $X$ is simple. This property is highly desirable for code generation. Furthermore, the constraints on $X$ expressed with a basis of cycles are useful to build the set of independent vectors needed for tiling. ${ }^{(16)}$

\subsection{Examples}

We illustrate our algorithm on two synthetic examples so as to illustrate the different cases that, at least in theory, may occur. Example 6

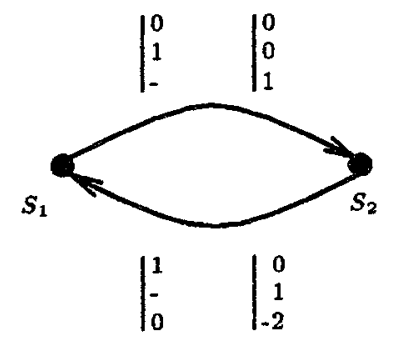

Fig. 20. RDG for Examplc 6. 


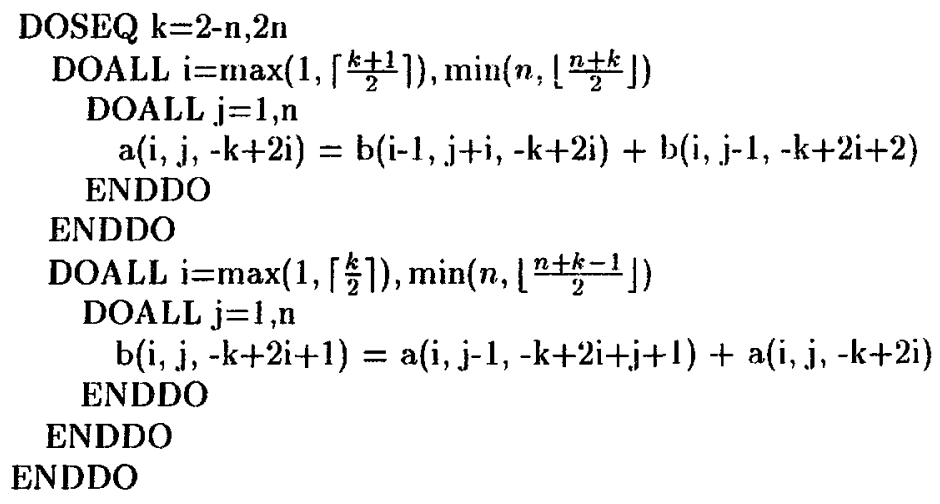

Fig. 21. Example 6, parallelized version.

can be scheduled with a single sequential loop, what Allen-Kennedy and Wolf-Lam fail to find. This example is presented with direction vectors. Example 7 is an example that cannot be parallelized successfully if dependences are approximated by direction vectors.

Example 6. (with direction vectors) Example 6 is the code of Fig. 19. Its reduced dependence graph, with direction vectors, is given in Fig. 20. In this example, the extension of Wolf-Lam with loop distribution proposed in Section 2 only finds one degree of parallelism, when one can

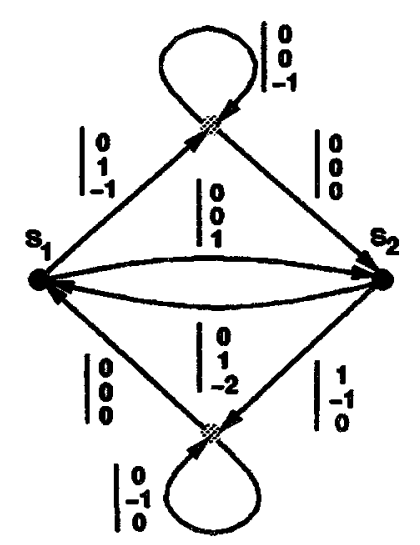

Fig. 22. Transformed RDG, Example 6. 


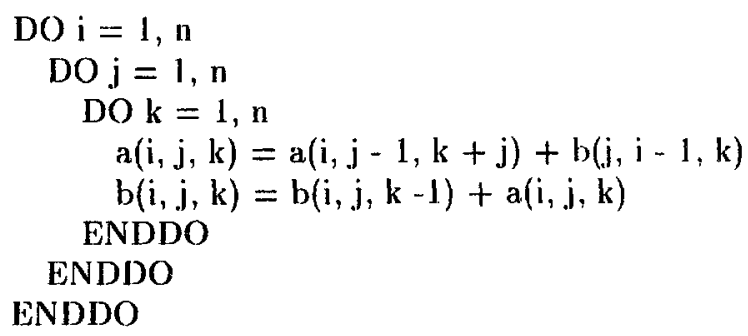

Fig. 23. Code of Example 7.

find two degrees. Indeed, for Wolf-Lam, because of the two dependences with a component equal to "- ", the first set of fully permutable loops only contains the $i$ loop. Only one dependence is removed from the RDG and the graph remains sirongly connected. The second set of fully permutable loops only contains the $j$ loop. Two dependences are removed and the graph is no more strongly connected. A loop distribution can be applied. Then the third loop is found to be parallel. Thus, Wolf-Lam finds one degree of parallelism.

We now apply our algorithm. The transformed RDG is given in Fig. 22. It has 4 vertices (two of them are virtual). The weights of elementary cycles are $(0,0,-1)$ and $(0,-1,0)$ for the self-loops and $(1,0,-1)$, $(1,-1,1),(0,2,-3)$, and $(0,1,-1)$ for the other elementary cycles. Therefore, one can find a one-dimensional schedule, for example $X=$ $(4,0,-2), \rho_{1}=0$ and $\rho_{2}=3$. Two degrees of parallelism can be exposed: the resulting code is given in Fig. 21.

Example 7. (with dependence polyhedra) We now illustrate our technique with the example of Fig. 23 in which the maximal parallelism can be detected only if dependences are approximated by a more accurate PRDG than a RDG labeled by direction vectors. Furthermore, both Allen-

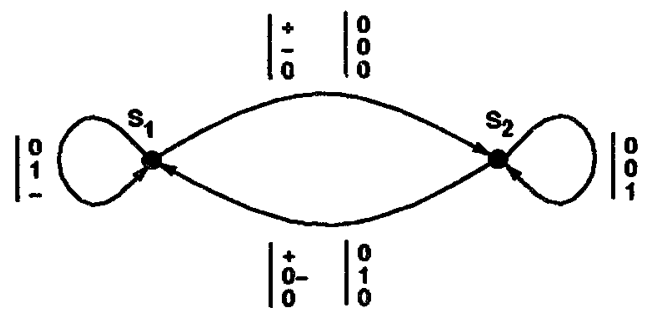

Fig. 24. RDG with direction vectors for Example 7. 
Darte and Vivien

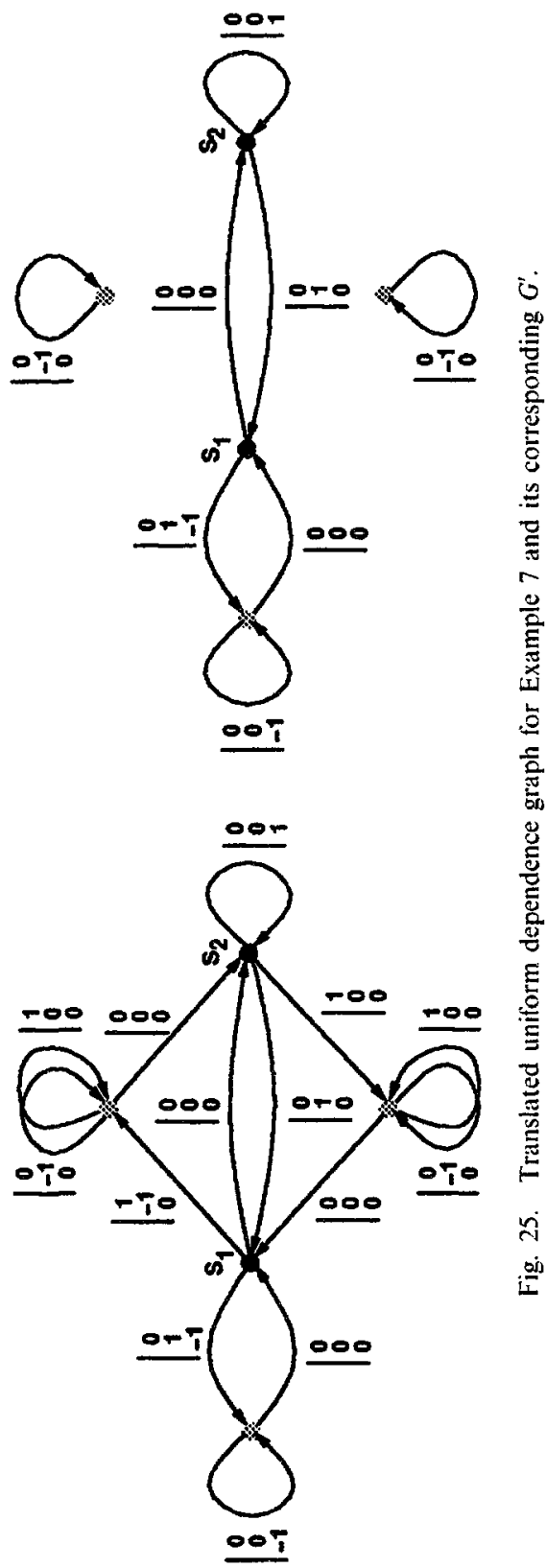


Kennedy and Wolf-Lam find that at least one of the statements must be fully sequentialized. With dependence polyhedra and our algorithm, the resulting $S_{1}$-latency is $O(N)$, the $S_{2}$-latency is $O\left(N^{2}\right)$, for a loop nest of depth 3 .

The graph $G_{i}$, depicted in Fig. 24 has been found by the dependence analyzer Tiny. ${ }^{135)}$

The uniformization step transforms $G_{o}$ into $G_{u}$ which is depicted in Fig. 25.

There is a multi-cycle of zero weight generated by all edges whose weight is orthogonal to $(1,0,0)$ (see Fig. 25). In $G^{\prime}$, the strongly connected component that contains $S_{1}$ and $S_{2}$ still has a multi-cycle of zero weight that visits an actual node $\left(S_{2}\right) . S_{1}$ is removed at depth 2 but $S_{2}$ is only removed at depth 3 . Thus, $S_{2}$ is purely sequential, whereas one degree of parallelism is detected for $S_{1}$. The multi-dimensional schedules are $(i, 2 j)$ for $S_{1}$ and $(i, 2 j+1, k)$ for $S_{2}$. The resulting code is therefore the code given Fig. 26.

Note that this is exactly what Allen and Kennedy's algorithm would find. However, if direction vectors are refined by more accurate dependence tests, one can find that the dependences can be approximated by the PRDG of Fig. 27. The reference to array $b$ generates indeed two dependences, a flow dependence whose dependence polyhedron has one vertex $(0,1,0)$ and one ray $(1,-1,0)$, and an anti dependence whose dependence polyhedron has one vertex $(1,-2,0)$ and the same ray than the flow dependence $(1,-1,0)$.

Note in Fig. 27 how this modification changes the structure of $G^{\prime} . S_{1}$ is now removed at depth 1 and $S_{2}$ at depth 2. For both statements, one more level of parallelism has been detected. The multi-dimensional schedules are $(4 i+2 j)$ for $S_{1}$ and $(4 i+2 j+1, k)$ for $S_{2}$. The resulting code is given in Fig. 28.

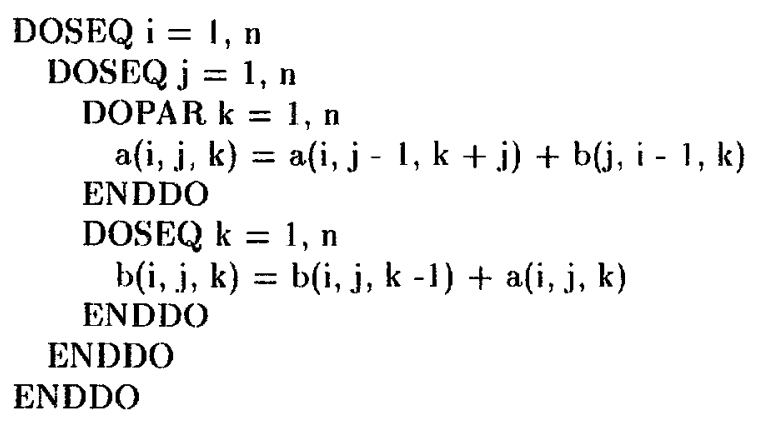

Fig. 26. Example 7, first parallelized version. 


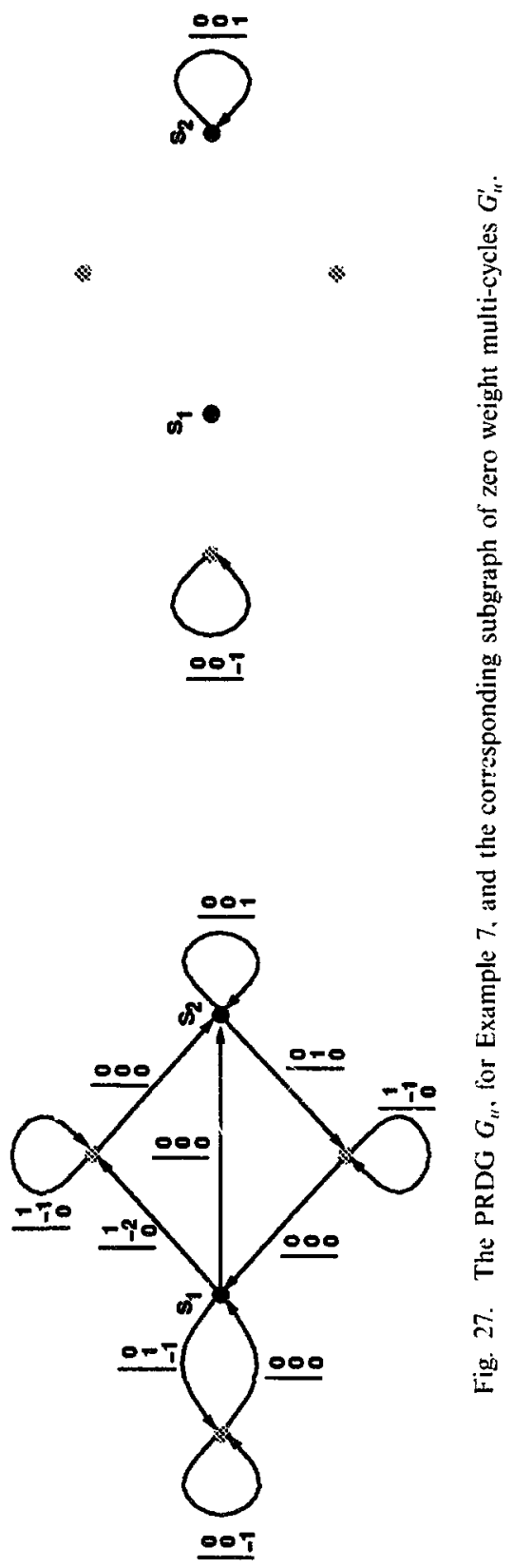




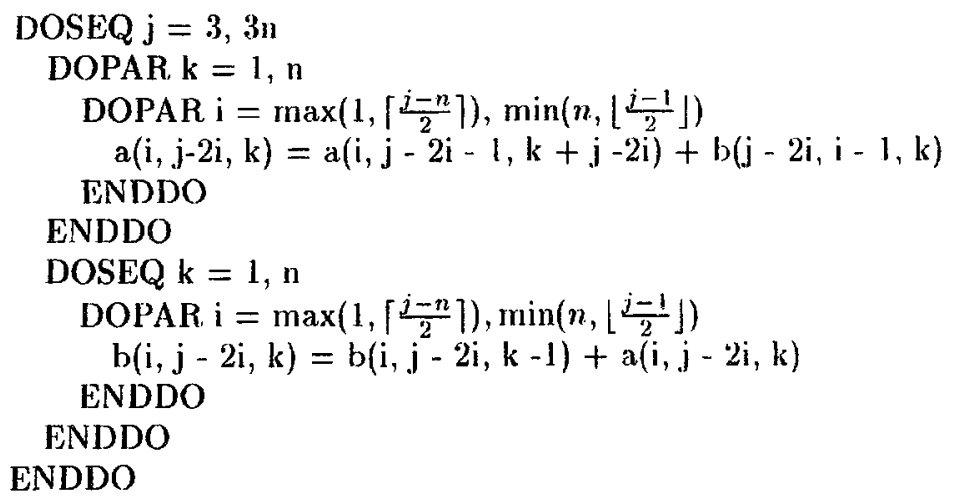

Fig. 28. Example 7, second parallelized version.

\subsection{Extension to Nonperfectly Nested Loops}

As proved in the previous sections, our scheduling algorithm is perfectly adapted to a description of distance vectors. When the loops are nonperfectly nested, the distance vector $J-I$ between two statements $S_{1}$ and $S_{2}$ is defined only for the first dimensions that correspond to common loops, i.e., loops that surround both $S_{1}$ and $S_{2}$.

Therefore, a natural way of extending the algorithm to nonperfect loop nests is to ignore, in each strongly connected component that appears during the decomposition, all dimensions that are not common dimensions. In other words, at a given depth of the algorithm, we truncate all vectors to the same dimensions ${ }^{6}$ and we apply on the truncated vectors the same technique as for perfectly nested loops. Finally, we complete each derived vector $X$ with ending zeros so that it fits the right dimension.

It turns out that this strategy remains nearly optimal, as long as no information is given on the noncommon dimensions. However, if at each level the code is nonperfectly nested then this algorithm is not more powerful than Allen and Kennedy's algorithm, since there is only one common dimension at each step.

To avoid this problem, we suggest another approach that enables to exploit the information on noncommon dimensions, and to benefit from the power of our algorithm for perfectly nested loops. As we did in Example 3, we first transform the code inio perfectly nested loops, by loop fusions or more complex techniques, possibly introducing "if" tests. Then,

\footnotetext{
"All vectors are truncated to the smallest dimension of a distance vector.
} 
the scheduling algorithm is applied on the transformed nest, reasoning on its dependence graph. Hereafter is an example, borrowed from the examples proposed in Petit, ${ }^{(25)}$ where the code is nonperfect at each level. Nevertheless, we point out that this technique is not general enough yet, and finding the adequate "perfectization" is not straightforward, if feasible...

Example 8. (nonperfect loop nest)

$$
\begin{array}{ll}
\text { DO } i=2, n & \\
s(i)=0 & \text { (Statement } S_{1} \text { ) } \\
\text { DO } j=1, i-1 & \\
s(i)=s(i)+a(j, i) b(j) & \text { (Statement } S_{2} \text { ) } \\
\text { ENDDO } & \\
\quad b(i)=b(i)-s(i) & \text { (Statement } S_{3} \text { ) } \\
\text { ENDDO } &
\end{array}
$$

In Example 8, the dependence graph has two strongly connected components, one with $S_{1}$, the other one with $S_{2}$ and $S_{3}$. We can thus apply a loop distribution to separate $S_{1}$ from $S_{2}$ and $S_{3}$. Furthermore, we integrate $S_{3}$ into the second loop, so as to obtain only perfect loops nests. We get the code of Fig. 29.

Applying our scheduling algorithm, we find that the vector $X=(x, y)$ has to satisfy the constraints $y \geqslant 1, x+y \geqslant 2, x \geqslant 0$, and $y \geqslant 0$. We find $X=(0,2)$ and $\rho_{S_{2}}=1$ and $\rho_{S_{3}}=0$ which corresponds to the code of Fig. 30 (once again without any effort to remove "if" tests).

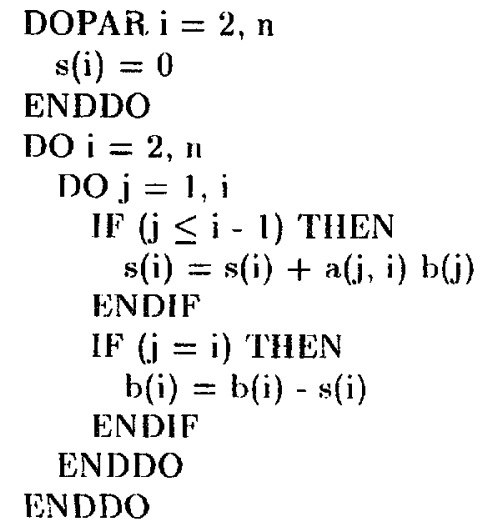

Fig. 29. Perfectly nested version of Example 8. 


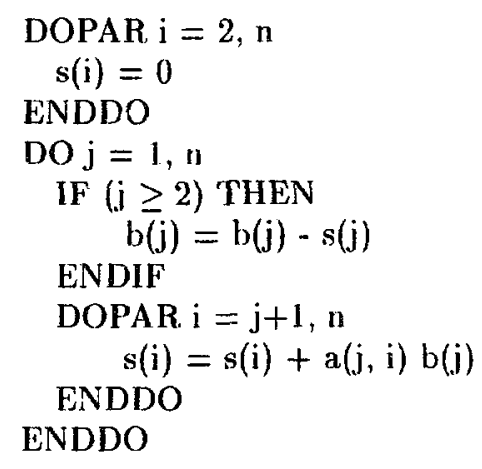

Fig. 30. Example 8, parallelized version.

\section{CONCLUSIONS}

We have presented an original scheduling algorithm to parallelize loops whose dependences are described through polyhedral reduced dependence graphs, i.e., reduced dependence graphs whose edges are labeled by an approximation of distance vectors by polyhedra. This representation of dependences is a generalization of direction vectors.

Our algorithm is nearly optimal, in the sense that it detects the maximal number of parallel loops that can be found, i.e., the minimal number of nested sequential loops (up to loop coalescing), as long as the only information available is the polyhedral reduced dependence graph. In particular, our algorithm is optimal for direction vectors, which generalizes Wolf and Lam's algorithm to the case of multiple statements.

We illustrated its power on several examples, examples with direction vectors as well as examples with more general polyhedral representations of distance vectors. All examples have been derived automatically with the algorithm we implemented and with the help of tools such as Tiny or Petit.

Our algorithm, like Feautrier's algorithm, was implemented at the Passau University, Germany, in the LooPo project. They compared the two algorithms on four codes. "1") Both parallelization algorithms output the same result, but our algorithm was quicker, and especially on the more complex codes (at least 10 times quicker on the three more complex codes). This experimentation does not prove that "the Darte-Vivien scheduler clearly outperforms the Feautrier scheduler on complex input programs," as it is written in Ref. 11. However, it shows that techniques as complex as Feautrier's ones are not always necessary to parallelize real codes. 
Some work remains for handling nonperfect loop nests. Our algorithm is indeed mainly well adapted for perfectly nested loops, or for common loops in nonperfect codes. However, to better exploit information on non common loops, a promising approach is to develop a method to transform nonperfect loop nests into perfect loop nests. This transformation remains to be fully automated.

Some work remains also to be done for exploiting the parallelism that has been detected. Indeed, a parallelizing algorithm only enhances operations that can be carried concurrently. It does not precise where these operations can be efficiently performed: automatic data mapping remains a crucial issue.

\section{ACKNOWLEDGMENTS}

We would like to thank the anonymous referees for their careful reading and helpful comments. We also wish to give special thanks to Paul Feautrier for many fruitful discussions that made clearer the link between the different parallelization algorithms, and to François Irigoin for his wise advice and his always pertinent remarks.

\section{REFERENCES}

1. David F. Bacon, Susan L. Graham, and Oliver J. Sharp, Compiler Transformations for High-Performance Computing ACM Computing Surrets 26(4):345-420 (1994).

2. John R. Allen and Ken Kennedy, Automatic Translation of Fortran Programs to Vector Form, ACM Trans. Program. Lang. S!s. 9(4):491-542 (October 1987).

3. Utpal Banerjee, A Theory of Loop Permutations, in D. Gelernter, A. Nicolau, and D. Padua. (eds.), Languages and Compilers for Parallel Computing. MIT Press. (1990).

4. Michael E. Wolf and Monica S. Lam, A Loop Transformation Theory and an Algorithm to Maximize Parallelism, IEEE Trans. Parallet Distribut. Sist. 2(4):452-471 (October $1991)$.

5. Wayne Kelly and William Pugh, A Framework for Unifying Reordering Transformations, Technical Report CS-TR-3193. University of Maryland (April 1993).

6. Paul Feautrier, Some Efficient Solutions to the Affine Scheduling Problem, Part II: MultiDimensional Time, IJPP 21(6):389-420 (December 1992).

7. R. M. Karp, R. E. Miller, and S. Winograd, The Organization of Computations for Uniform Recurrence Equations, $J$. ACM 14(3):563-590 (July 1967).

8. Alain Darte and Frédéric Vivien, A Classification of Nested Loops Parallelization Algorithms. INRIA-IEEE Symp. on Emerging Tedhologies and Fuctory Autonoton IEEE Computer Society Press, pp. 217-224 (1995). Will also appear in PPL. Special issue (1997).

9. Pierre-Yves Calland. Alain Darte. Yves Robert. and Frédéric Vivien. Plugging Anti and Output Dependence Removal Techniques into Loop Parallelization Algorithms. Parallel Computing 231 1, 2):251-266 (1997). 
10. Alain Darte, Georges-André Silber, and Frédéric Vivien, Combining Retiming and Scheduling Techniques for Loop Parallelization and Loop Tiling. Parallel Processing Lethers (1997). Special issue. to appear. Also available as Technical Report L.IP. ENS. Lyon, RR96-34.

11. Wolfgang Meisl. Practical Methods for Scheduling and Allocation in the Polytope Model. World Wide Web document. URL:http: brahms.fmi.uni-passiaude cl loopo doc.

12. Leslic Lamport. The Patallel Execution of DO Loops. Commen. AC:M 17(2):83-93, (February 1974).

13. Alain Darte and Yves Robert, Constructive Methods for Scheduling Uniform Loop Nests. IEEE Truns. Purullel Distrihut. Sist. 5(8):814-822 (1994).

14. Alatin Darte and Yves Robert, Alline-by-Statement Scheduling of Uniform and Aline Loop Nests over Parametric Domains. J. Parallel and Distrihuted Computing 29:43-59 (1995).

15. Paul Feautrier. Some Ellicient Solutions to the Affine Scheduling Problem. Part 1: OneDimensional Time. IIPP 21(5):313-348 (October 1992).

16. Amy W. Lim and Monica S. Lam. Maximizing Paralledism and Minimizing Synchronization with Aline Transforms, Proc. 24h Am. ACM S/GPL.AN-SIGAC'T Simp. Primciples of Progr. Lang. (January 1997).

17. Alain Darte. Leonid Khachiyan, and Yves Robert. Lincar Scheduling is Nearly Optimal. Parallel Processing Lethers 1(2):73-81 (1991).

18. Patrick Le Gotëstier d Argence. An Asymptotically Optimal Alfine Schedule on Bounded Convex Polyhedric Domains, Proc. Euro-Par ${ }^{96}$ Parallel Processing. Vol. 1124 of LNCS. Springer-Verlag ( August 1996).

19. Paul Feautrier. Diltallow Analysis of Arraty and Scalar References. Int. JPP 20(1):23-51 (1991).

20. Jean-François Collard. Denis Barthou. and Patul Feattricr. Fužy Array Datallow Analysis. Proc. 5th ACM SIGPLAN Symp. an Principles and Practice of Parallel Programming. Santa Barbara, California (July 1995).

21. Altin Darte and Fréderic Vivien. On the Optimality of Allen and Kennedys Algorithm for Parallelism Extraction in Nested Loops. Journal of Parallel Algorithms and Applications 12(1 3):83-112 11997). Special issuc on Optimizing Compilers for Parallel Languages.

22. Alain Darte and Frederic Vivien. Revisiting the Decomposition of Karp. Miller. and Winograd. Paralled Processing Lenters 5(4):551-562 (December 1495).

23. Giene H. Golub and Charles F. Van Loan. Marrix Compurutions. Johns Hopkins. Second Edition (1989).

24. Jack J. Dongarra and Stanky C. Eisenstat, lud. World Wide Web document. URL: hup://netlib.bell-labs.com/netlib/benchmark/index. html.

25. W. Kelly, V. Maslov. W. P'ugh, E. Rosser. T. Shpeisman, and D. Wonnacott. New User Interface for Pesit and Olher Interfates: Liser Guide. University of Matryland (June 19951.

26. Arthur J. Bernstein. Analysis of Programs for Parallel Processing. IEEE Trans. Elsctronic (imputers 15:757-762 (October 1966).

27. John R. Allen and Ken Kennedy, PFC: $\Lambda$ program to convert fortran to Parallel Form. Techrical Report MASC-TR82-6. Rice University. Houston. Texas. (1982).

28. Michacl Wolfe. Optimizing Supereompilers for Supercomputers Ph.D. Thesis. Department of Computer Science. University of Illinois at Urbana-Champaign (October 19821.

29. Michael Wolfe. Optimizing Supercompilers for Supersomputers. MIT Press. Cambridge Massachusettes (1989).

30. François Irigoin and Rémy Triolet, Computing Dependence Direction Vectors and Dependence Cones with Linear Systems, Technical Report ENSMP-CA1-87-E94. École des Mines de Paris. Fontainebleau. France (1987). 
31. François Irigoin and Rémy Triolet, Supernode Partitioning, Proc 15th Amm. ACM Symp. Principles of Progr. Lang., San Diego, California, pp. 319-329 (January 1988).

32. François Irigoin. Pierre Jouvelot, and Rémy Triolet, Semantical Interprocedural Parallelization: An overview of the PIPS Project, Proc. ACM ht. Conf. Supercomputing. Cologne, Germany (June 1991).

33. Alexander Schrijver, Theory of Linear and Integer Programmeng, John Wiley and Sons, New York (1986).

34. François Irigoin and Rémy Triolet. Dependence Approximation and Global Parallel Code Generation for Nested Loops, Proc. Int. Workshop on Purallel and Distributed Algorithms (October 1988).

35. Michael Wolfe, TINY, a Loop Restruchuing Research Tool, Oregon Graduate Institute of Science and Technology (December 1990).

36. Alain Darte and Frédéric Vivien. Optimal Fine and Mediun Grain Parallelism Detection in Polyhedral Reduced Dependence Graphs, Technical Report 96-06. LIP. ENS-Lyon. France (April 1996).

37. Sailesh K. Rao, Regular Iterative Algorithms and their Implementations on Processor Arrays. Ph.D. Thesis, Stanford University (October 1985).

38. Vwani P. Roychowdhury. Derivation, Extensions and Parallel Implementation of Regular Iterative Algorithms, Ph.D. Thesis, Stanford University, December 1988.

39. S. Rao Kosaraju and Gregory F. Sullivan. Detecting Cycles in Dynamic Graphs in Polynomial Time (preliminary version), Proc. 20th Ann. ACM Simpos. Theory of Computing. pp. 398-406 (May 1988).

40. Alain Darte and Frédéric Vivien, Automatic Parallelization based on Multi-Dimensional Scheduling. Technical Report 94-24, LIP. ENS-Lyon, France (September 1994).

41. M. Gondran and M. Minoux, Graphs and Algorithms. John Wiley and Sons (1984).

42. Thomas H. Cormen, Charles E. Leiserson, and Ronald L. Rivest, Introduction to Algorithms. MIT Press (1990). 